\title{
Tous les comptes rendus au format PDF
}

\section{(2) OpenEdition \\ 1 Journals}

Édition électronique

URL : https://journals.openedition.org/ahrf/4143

DOI : 10.4000/ahrf.4143

ISSN : 1952-403X

Éditeur :

Armand Colin, Société des études robespierristes

Édition imprimée

Date de publication : 1 décembre 2002

ISSN : 0003-4436

Référence électronique

"Tous les comptes rendus au format PDF ", Annales historiques de la Révolution française [En ligne], 330 | octobre-décembre 2002, mis en ligne le 17 avril 2008, consulté le 23 avril 2022. URL : http:// journals.openedition.org/ahrf/4143; DOI : https://doi.org/10.4000/ahrf.4143 


\section{COMPTES RENDUS}

Alain BLONDY, L'Ordre de Malte au XVIIIe siècle. Des dernières splendeurs à la ruine, Paris, Éditions Bouchene, 2002, 523 p.

Cet ouvrage, de facture très traditionnelle, narre par le menu la fin peu glorieuse de l'Ordre de Malte qui vécut ses dernières belles années sous le Grand Maître Pinto (1741-1773), dont les prétentions absolutistes et monarchiques se heurtèrent à la faiblesse des moyens financiers. Les impositions des commanderies n'avaient pas été réévaluées depuis le XVje sjècle, les États européens répugnaient à des sorties d'argent à destination de l'île ; la place conquise par Malte dans le commerce méditerranéen profitait plus à la bourgeoisie maltaise qu'à l'Ordre. Quant à la politique étrangère d'un Ordre devenu un véritable État, dont l'activité militaire était en total déclin, elle était étroitement subordonnée aux États bourbons, liés par le pacte de Famille, qui avaient fait de la Méditerranée occidentale un lac intérieur; Malte demeurait un fief de la couronne de Sicile, les États bourbons fournissaient $77 \%$ des revenus et les trois Langues françaises (Provence, Auvergne et France) la moitié. La politique étrangère de l'Ordre se faisait essentiellement à Paris, ce qui ne laissait pas de provoquer quelques difficultés occasionnelles puisque la France demeurait alliée à l'Empire ottoman. Le second successeur de Pinto, Emmanuel de Rohan, élu en novembre 1775, réunit un chapitre général en 1776 (il ne l'avait pas été depuis 1631) et réussit à imposer quelques réformes qui redonnèrent à l'Ordre une fragile santé financière. Il promut une politique de récupération de biens, parvint à réformer la justice (le Suprême Magistrat de Judicature), promulgua un code inspiré des idées des Lumières. Une Langue bavaroise, soutenue par la France, s'ajouta aux huit précédentes, alimentée par les biens des jésuites; il s'agissait de faire contre-poids, dans l'aire allemande, aux Habsbourg, aux Hohenzollern et aux Hanovre. À la veille de la Révolution, le jeu fut quelque peu troublé par les ambitions russes en Méditerranée et les manœuvres de l'ambassadeur Psaro, envoyé de Catherine II à Malte, par la politique de la reine Marie-Caroline de Naples, fille de l'impératrice Marie-Thérèse, qui s'éloignait des cours des Bourbons, par les rivalités nationales de plus en plus vives chez les chevaliers.

L'Ordre fut confronté dès 1789 aux nouveaux cours des choses : il perdit dès le mois d'août les revenus de ses dîmes françaises; Camus, janséniste et constituant, futur premier archiviste de France, voulut déclarer les propriétés de l'Ordre, comme celles du clergé, biens nationaux; seul le statut de puissance étrangère que Malte s'était fait reconnaître, permit la sauvegarde des biens jusqu'en 1792. Si quelques chevaliers comme Ransigat, Dolomieu ou Fay étaient favorables à la Révolution, le Grand Maître 
Rohan lui était tout à fait hostile; il tâcha néanmoins de faire prévaloir une politique de neutralité et de conciliation, tout en recherchant des appuis du côté de l'Angleterre et surtout de la Russie. Le Directoire et son ministre Delacroix voulaient surtout protéger les intérêts commerciaux français à Malte et empêcher que l'île ne tombât sous l'influence anglaise. Rohan mourut en 1797 et fut remplacé par Ferdinand de Hompesch, un Allemand par lequel l'Ordre espérait s'attirer la protection de l'Empereur. Il se trouva confronté à une grave crise financière - il fallait entretenir les chevaliers français, privés de leurs revenus - et à la désorganisation des circuits commerciaux qui mécontentait les milieux d'affaires. Par ailleurs, le Second Directoire, Talleyrand et Bonaparte intégrèrent Malte, clé du Levant, dans une stratégie méditerranéenne qui allait de l'Italie à l'Empire ottoman. Dès 1797, Bonaparte séquestra les commanderies d'Italie. L'expédition d'Égypte fit de la prise de Malte le prélude des opérations futures; en juin 1798 l'île tomba dans les mains des Français à la première sommation et par la convention du 12, l'Ordre renonça au profit de la France à sa souveraineté. Ni les chevaliers divisés entre nations et par des choix idéologiques depuis 1789 , ni la noblesse locale éloignée du gouvernement par l'Ordre, ni la bourgeoisie désemparée par le marasme économique, ni le peuple maltais méprisé et souffrant n'offrirent une vraie résistance. Hompesch se réfugia à Trieste où il ne fut que très médiocrement soutenu par François II qui ne voulait pas s'aliéner la Russie, au moment où elle prenait la tête d'une coalition contre la France. Car le Grand Prieuré de Russie, très hostile à Hompesch accusé de passivité, réussit une véritable O.P.A. sur l'Ordre en proclamant en octobre 1798 l'empereur Paul Ier Grand Maître.

La flotte française quitta Malte dès le 19 juin. Sous l'égide de la République française, le pouvoir passa à la bourgeoisie commerçante de La Valette. Les biens de l'Ordre devinrent biens nationaux, nombre de couvents furent supprimés et les religieux regroupés, l'esclavage fut aboli, la citoyenneté proclamée. Un Journal de Malte, en français, devait conforter le Nouveau Régime. Mais très rapidement, les communications entre Malte et le Directoire furent interrompues par le blocus anglais qui amena dans l'île la pénurie et sa conséquence, l'insécurité. Parallèlement naissait un mouvement de patriotes nationalistes. À propos d'une confiscation au couvent des carmes de Mdina, une révolte éclata, qui souleva les campagnes pauvres et très attachées à la religion, contre les Français et leurs soutiens maltais urbains et cultivés. Les Français ne conservèrent que $\mathrm{La}$ Valette et les Trois Cités. Le blocus se renforça lorsque Nelson revint d'Aboukir en octobre et prit l'île de Gozo. Après 19 mois, la reddition de Malte fut conclue le 4 septembre 1800 entre les Français et les Anglais, sans que les Maltais ne fussent consultés. L'occupation anglaise remplaça la française. En dépit de la paix d'Amiens qui rendit l'île à l'Ordre, sous la garantie des grandes puissances, les Anglais demeurèrent à Malte où ils furent mieux supportés que les Français par une population chez qui la longue détestation des chevaliers français d'Ancien Régime avait été remplacée par l'hostilité à l'anticléricalisme révolutionnaire. En 1813, les Maltais furent déclarés par Londres sujets de la Couronne britannique, décision qui fut entérinée par le traité de Paris et le congrès de Vienne. Ainsi prenait fin le lien, qui datait de 1530, entre Malte, devenu au cours des ans un pôle économique important en Méditerranée, et l'Ordre qui avait perdu depuis longtemps sa vocation initiale.

Nous avons donc là un récit méticuleux des derniers temps de l'Ordre, appuyé sur de considérables dépouillements d'archives diplomatiques. L'Ordre et l'île de Malte avaient longtemps subsisté l'un par l'autre. Au XVIIIe siècle, il est certain que les intérêts des Maltais, quelle que soit leur catégorie sociale, y compris le clergé local, et ceux des chevaliers étaient de plus en plus divergents. Une histoire de l'Ordre ne peut être dissociée d'une histoire sociale et économique de Malte, assez peu présente dans cet ouvrage.

Claude MICHAUD 
Antoine-Marie Graziani, Pascal Paoli, Père de la patrie corse, Paris, Tallandier, coll. Figures de proue, 2002, $340 \mathrm{p}$.

Le matraquage médiatique auquel nous sommes soumis ne saurait nous abuser, ce livre ne relève pas de l'enquête historique.

Quelques exemples faciles à vérifier suffiront à s'en convaincre. Je les emprunterai à la période révolutionnaire, et plus particulièrement à celle qui va de la réunion des États généraux à l'insurrection fédéraliste de 1793, qui est à la base même de tous mes travaux.

Sur 43 pages ( 1634 lignes) que l'auteur lui consacre (pp. 275-317), 14 pages (515 lignes) sont occupées par des citations. Sur ce nombre, 6 pages ont été purement et simplement recopiées dans mes travaux :

1. La Corse française - 30 novembre 1789-15 juin 1794, thèse de Doctorat en Histoire, Société des études robespierristes, Bibliothèque d'Histoire révolutionnaire, Paris, 1980, $224 \mathrm{p}$.

2. "Paoli et les frères Bonaparte - De la légende à l'Histoire", colloque Napoléon : Problèmes d'histoire de la Corse (de l'Ancien Régime à 1815), Société des études robespierristes et Société d'Histoire Moderne, actes du Colloque d'Ajaccio (1969), Paris, 1971, pp. 131-145.

3. «La mort du roi », Colloque : Saint-Denis ou le jugement dernier des rois, Univ. de Paris VIII, Institut d'Histoire de la Révolution française, Paris-Sorbonne, et Comité du Bicentenaire de la Révolution françaisé, Éditions de l'Espace Européen, 1992, pp. 265-272.

4. La jeunesse de Napoléon - les dessous de l'Histoire, Paris, Lettrage Distribution, 2001,384 p.

Je n'irai pas jusqu'à l'accuser de plagiat. Je noterai néanmoins qu'emporté par son élan, l'auteur a recopié des centaines d'autres lignes de ma thèse, en oubliant de le signaler. Voir, à titre d'exemple, les pages 103 et 104 de ma thèse La Corse française et les pages 298 et 299 de son livre où l'on trouve 32 lignes rigoureusement identiques.

Les autres citations, et les commentaires qui les accompagnent, ne sont que digressions faites pour brouiller les pistes et nous entraîner loin, très loin de la vérité. Voir, à titre d'exemple, l'accent qu'il met sur le témoignage du conventionnel Moltedo qui, en 1796, accusa son collègue Saliceti d'avoir acheté le plus beau domaine de la Corse, en faisant "emprisonner... des concurrents potentiels pour les écarter de l'adjudication et pour en être le seul acquéreur à bas prix» (p. 296 ), «d'avoir intrigué auprès de ses comités pour être envoyé commissaire en Corse» (sic) (p. 306), d'avoir violé le secret des postes, etc., en oubliant :

1) que toutes ces accusations avaient été portées trois années auparavant par le département de la Corse. Voir lettre du Conseil général aux commissaires Lacombe Saint-Michel et Delcher, du 15 mai 1793;

2) que Saliceti avait riposté en accusant le susdit département de rébellion. Voir réponse du citoyen Saliceti, imprimée et publiée en Corse, le 14 juin dernier, aux faits calomnieux contenus dans la lettre du 15 mai demier, écrite par les membres du département de Corse rebelles, et actuellement en état d'accusation, aux citoyens Delcher et Lacombe SaintMichel, représentants du peuple, qui l'ont méprisée, et que Constantini, soi-disant député extraordinaire dudit département, a fait réimprimer et distribuer le 9 de ce mois, six jours après le départ de Saliceti pour sa mission;

3) que Moltedo avait soutenu son collègue ;

4) que le témoin cité à la barre de l'Histoire était l'un des trois députés «infidèles " que la Consulta, réunie à Corte, du 27 au 29 mai 1793, avait dénoncés à la Convention. Voir P.V. de la réunion, plus particulièrement les articles arrêtés par 
l'assemblée générale des mandataires du peuple corse, 27 au 29 mai 1793. Et pour tout dire, l'un des administrateurs corrompus qui, pour sauver leur tête, n'avait pas hésité à tourner toutes les forces de la république contre leur île natale, l'un des responsables des flots de sang versés en Corse au cours de cette sombre période (toutes les pièces cidessus mentionnées sont citées dans ma thèse La Corse française...).

Et que dire des libertés que l'auteur prend avec la chronologie ? À la page 281, par exemple, où l'on peut lire : «Paoli arrive le 3 avril 1790 à Paris, escorté de ses compagnons, qui ont été rejoints par Chiappe, Peraldi et Moltedo, délégués par Ajaccio». Oui, que dire lorsqu'on sait que ces trois députés - qui en réalité étaient quatre, comme les « trois mousquetaires », A. Graziani oubliant l'abbé Peretti - nommé le 9 avril - voir le compte rendu de l'assemblée d'Ajaccio dans le Giornale Patriottico di Corsica du 24 avril 1790 (cité dans mon livre La jeunesse de Napoléon - les dessous de l'Histoire, p. 130), ne quittèrent la Corse que le 16 mai et ne purent de ce fait même arriver à Paris au plus tôt que deux mois après les faits relatés.

Je n'ai trouvé qu'un moyen d'éviter les pièges tendus sous nos pas, classer les événements de la période susdite en trois catégories :

1) ceux qui apportent de l'eau au moulin d'A. Graziani qui les reprend purement et simplement à son compte, en reconnaissant parfois ce que son livre doit à mes travaux, notamment lorsque ces derniers sentent un peu trop le soufre et qu'il n'est pas mauvais pour lui de s'abriter derrière un scientifique reconnu. «En résumé, [lit-on à la p. 275] la Corse n'est peut-être pas ce "pays où les tigres régnaient" que décrit le jeune lieutenant Bonaparte, mais, certainement comme le note Jean Defranceschi "une terre de conquête où il ne faisait pas bon vivre pour ceux qui [n'appartiennent] pas à la race des conquérants"» (sic);

2) ceux qui prêtent à discussion et que l'auteur reprend également à son compte, mais en donnant aux sources le coup de pouce nécessaire pour faire basculer la vérité dans le sens voulu. Ce qu'il dit sur l'envoi par l'assemblée de Bastia d'une députation chargée de ramener Paoli dans sa patrie : «elle est composée de Murati, Belgodere, Casabianca et Panattieri. Seuls les deux premiers peuvent remplir leur mission, à leurs frais d'ailleurs... » (p. 281) est vrai, à un détail près : l'ordre nominal des quatre députés. "Le choix tomba sur MM. Casabianca, Panattieri, Morati et Belgodere; les deux premiers seulement se sont rendus à Paris, MM. Morati et Belgodere ayant été forcés par une maladie de rester en Corse,» lit-on dans les Discours prononcés à l'Assemblée nationale par le général Paoli, les députés extraordinaires de Corse; et la réponse de $M$. le Marquis De Bonnay, président de l'Assemblée nationale, Paris chez Garnéry, l'an premier de La Liberté.

Sous-titre : détails historiques sur la présentation des députés extraordinaires de Corse et du général Paoli à l'Assemblée nationale et au Roi, faite le 22 avril 1790. Publiés dans mon livre La Corse et la Révolution française - recueil de conférences faites, tant en France qu'à l'étranger, à l'occasion du Bicentenaire de la Révolution française, (Ajaccio, Éditions Cyrnos et Méditerranée, 1991, 147 p.), p. 113, lignes 11-17.

Un détail qui lui permet, à la page suivante, de passer à la trappe F. Panattieri, celui qui conduisit la délégation de Corse, et par voie de conséquence, le discours particulièrement instructif qu'il prononça à l'Assemblée nationale, le 22 avril 1790. En voici la teneur :

"Messieurs, La Corse libre nous députe vers vous pour vous rendre graces de l'avoir affranchie. Le despotisme nous avoit accablés; mais, nous osons le dire, il ne nous avoit pas soumis. Votre justice seule nous a conquis, et c'est à votre générosité que nous rendons les armes : nous haïssions des maîtres dans le nom français, nous y bénissons des libérateurs et des frères. Pendant quatre cents ans nous avons combattu pour la liberté, nous avons versé des flots de sang pour elle, et nous n'avons pu l'obtenir; dans un jour vous nous l'avez donnée, voyez si nous 
pourrions être ingrats et rebelles? Toute l'Europe admire vos travaux, toute la France vous remercie de vos lois; mais il n'est pas de département qui les admire plus et en sente mieux le prix que la Corse. Il n'est pas un de vos décrets qui n'ait rétabli un droit, brisé une entrave, donné un bienfait au peuple : jugez si ses sentimens sont variables?

En comparant notre état passé avec ce que nous avons été, ce que nous allons être, tout accroit notre gratitude et cimente notre attachement.

Nous étions une nation faible, un état borné; devenus Français, nous sommes une nation puissante, nous avons la force d'un grand empire; il devient le garant de notre tranquillité, et nous sommes pour lui un fort de défense et de commerce.

Le fondement solide de toute union, l'avantage mutuel, garantit la nôtre. Oui, Messieurs, soyez en certains, la France n'a point de peuple plus dévoué, l'Assemblée nationale de sectateurs plus zélés, la Constitution et le Roi de sujets plus fidèles que le peuple corse.

Ce tableau de nos devoirs en retrace un à nos cœurs d'autant plus cher, que vous-mêmes l'avez consacré, et qu'il tient aux sentimens que nous vous offrons.

En vous parlant de reconnoissance, nos compatriotes n'ont pu manquer de se rappeler, parmi ceux qui ont combattu pour la défense de leur liberté, l'homme qui leur en peignoit si vivement les charmes; et par un sentiment de justice, ils ont voulu que celui qu'ils eurent à leur tête dans leur infortune, fut à leur tête en ce jour de triomphe et de félicité.

Ce devoir aquitté, ils le redemandent avec instances, pour avoir sous leurs yeux l'exemple de ses vertus, pour profiter de ses lumières dans l'observation des lois que vous faites, pour les aider à réparer les maux d'un régime oppresseur qui a tout dévasté.

Cette dernière pensée, en nous affligeant sur l'état de notre patrie, nous porte vers une autre qui nous console : en venant des extrémités de la France, et la traversant presque entière, nous avons vu par-tout le spectacle de la joie et de la prospérité, et par-tout nous avons entendu en attribuer le bienfait à l'Assemblée nationale; par-tout nous avons entendu bénir sa sagesse, louer son ouvrage; et quand nous vous apportons l'hommage de notre gratitude et de notre respect, ce n'est pas celui de la Corse seule, c'est celui de toute la France devenue notre patrie commune » (Ibid., pp. 114-116).

Et comme si cela était insuffisant d'amputer le discours de Paoli des passages embarrassants. Je le reconstitue donc en signalant par des caractères gras la partie manquante dans le livre en question :

"Ce jour est le plus heureux et le plus beau de ma vie. Je l'ai passée à rechercher la liberté et j'en vois ici le plus noble spectacle. J'ai quitté ma patrie asservie; je la retrouve libre, je n'ai plus rien à désirer.

Je ne sais depuis une absence de vingt ans quel changement l'oppression aura fait sur mes compatriotes; il n'a pu être que funeste, car l'oppression ne fait qu'avilir; mais vous venez d'ôter aux Corses leurs fers, vous leur avez rendu leur vertu première.

En retournant dans ma patrie, mes sentiments ne peuvent vous être douteux. Vous avez été généreux pour moi, et jamais je n'ai été esclave. Ma conduite passée, que vous avez honorée de votre suffrage, vous rêpond de ma conduite future : j'ose dire que ma vie entière a été un serment à la liberté, c'est déjà l'avoir fait à la Constitution que vous établissez; mais il me reste à le faire à la Nation qui m'adopte et au Souverain que je reconnois; c'est la faveur que je demande à l'auguste Assemblée ». (Comparer Graziani p. 282, et Defranceschi, La Corse et la Révolution française, p. 118). 
Un détail qui ne l'empêche pas, lors de la réception de Paoli par les amis de la Constitution, de ramener F. Panattieri à la surface (p. 283), de recommencer lors de la présentation au roi, le 30 avril (p. 284), et ainsi de suite.

3) ceux franchement incompatibles avec sa "vérité » et qu'il passe purement et simplement sous silence. Voir, à titre d'exemple, le sort réservé à l'assemblée électorale tenue à Corte du 12 au 23 septembre 1792, et, avec elle, à la tentative pour renverser l'administration en place et séparer la Corse de la France. Autrement dit à l'intervention de Paoli qui fit échouer le coup en disant « qu'il n'avait jamais été d'avis que l'on dît aucune innovation ni que l'on procédât à aucun changement, soit dans l'administration supérieure soit dans les autres corps légalement constitués; que ce système désorganisateur était bien éloigné de ses principes, qu'il n'y avait que les ennemis du bien public qui avaient pu concevoir ces idées désastreuses qui tendraient à séparer la Corse de la mère patrie à laquelle elle devait restée attachée par reconnaissance pour tous les bienfaits qu'elle avait versés sur elle... » [sic]. Voir la relation que j'en fais dans La Corse française..., p. 100, avec à l'appui le texte intégral du P.V. de la réunion. Ibid., p. 197 et suivantes.

Trois catégories qui permettent de voir comment A. Graziani parvient à faire jouer à Paoli un rôle bien différent de celui qui fut alors le sien.

Le reste de l'ouvrage est "du même tonneau". Cette biographie du père de la patrie corse ne contient rien que nous ne sachions déjà, rien sur l'enfance corse de Pascal Paoli, rien sur sa jeunesse napolitaine, rien sur son généralat, rien sur son exil anglais, rien sur l'éphémère Royaume anglo-corse et les dernières années du héros et martyr de la liberté ; bref rien qui ne soit consigné dans les travaux de l'un ou l'autre de ses prédécesseurs, savoir des érudits qui, depuis 156 ans, c'est-à-dire depuis la publication des Lettere di Pasquale Paoli par Nicolo Tommaseo (Firenze 1846), ont consacré leur vie à recueillir, classer, annoter et publier des milliers de documents, et naturellement des universitaires qui ont consacré la leur à séparer le vrai du faux. Il ne peut être question de les citer tous ici. Les trois derniers suffiront :

John M. P. Mc Erlean, professeur à la York University à Toronto (Canada). Croira-t-on qu'il n'y a dans sa thèse sur l'un des personnages clefs de la période, C. A. Pozzo di Borgo, et ses multiples communications sur Paoli, le Royaume anglocorse, P. Masseria, Bonaparte, etc. publiées dans des revues prestigieuses comme les Annales historiques de la Révolution française, la Revue de l'Institut Napoléon, les Proceedings du Consortium on Revolutionary Europe, etc., rien qui vaille le détour? Pas même sa communication à la Fondation Paoli sur « Le Capitaine Charles Wood et l'évasion du Général Paoli de Corse en juin 1769 " publiée dans le Bulletin de la Société des Sciences historiques et naturelles de la Corse, $n^{\circ} 663,1993, \mathrm{pp} .9-17$ ?

Marie-Thérèse Avon-Soletti, maître de conférences à l'Université de Saint-Étienne. Croira-t-on qu'il n'y a dans sa thèse d'État : La Corse et Pascal Paoli - Essai sur la constitution de la Corse (soutenue à Lyon III, publiée à La Marge Édition, Ajaccio 1999, avec le concours de la Collectivité territoriale de Corse) pas une ligne qui mérite de retenir l'attention?

Charles Napoléon que l'on ne présente pas. Croira-t-on qu'il n'y a dans son livre Bonaparte et Paoli - Aux origines de la question corse (Perrin, 2000) pas un mot méritant le moindre commentaire? Non bien sûr ! Mais alors pourquoi ce silence sur ces publications qui, toutes réunies, représentent des centaines d'années de recherche, dans lesquelles A. Graziani n'a eu qu'à se servir? Et comment ne pas s'interroger sur les raisons qui ont conduit le dernier maillon d'une longue chaîne d'intellectuels à s'approprier jusqu'aux miettes des travaux de tous les autres?

Voir, à titre d'exemple, la relation qu'il fait de la Consulta du 22 mai 1768 où l'auteur nous apprend que le fameux appel à la valorosa Giuventu di Corsica n'a pas été lancé par Charles Bonaparte, comme on l'a si souvent écrit, mais par Pascal Paoli 
(p. 231), en oubliant simplement de nous dire que la découverte n'est pas de lui, mais du signataire de ce papier (voir ma communication à l'Institut Napoléon : "Charles Bonaparte face à la conquête française de la Corse " publiée dans la Revue de l'Institut Napoléon, $\mathrm{n}^{\circ} 152$, 1989-I, pp. 11-17, et mon article «Paoli» dans le Dictionnaire Napoléon, sous la direction de Jean Tulard, Paris, Fayard 1987, pp. 1295 et suiv.).

\section{Jean DEFRANCESCHI}

Mémoires (1760-1820) de Jean-Balthazar de Bonardi du Ménil, gentilhomme normand, Paris, Honoré Champion, 2001, 904 p.

Il s'agit d'une riche entreprise mémoriale et autobiographique, Jean-Jacques étant son maître, l'auteur, Bonardi du Ménil, n'a qu'un souci, qu'une volonté celle de se mettre, lui exclusivement, en scène. Cet ouvrage, édité et annoté par Claude Hohl a été préfacé par André Corvisier.

Lintérêt premier de cette entreprise c'est que la vie de Bonardi couvre l'ensemble de la période de transition. Celui-ci a vécu les «douceurs » de l'Ancien Régime, l'événement Révolution dont il défendit, contre son milieu, les acquis principaux, il a assisté au sacre de Napoléon, puis au retour des Bourbons. À l'évidence, il n'est pas le seul à avoir ce parcours, à suivre un si long itinéraire mais l'originalité du récit tient au fait qu'il ne fut ni acteur, ni opposant, il est au spectacle, il décrit de telle sorte qu'il devient un miroir de son époque. Ses réelles préoccupations demeurent familiales, quotidiennes et plus que tout personnelles. Il ne voit et ne raconte les événements que par rapport à son "ego" qu'il ne cesse un instant de flatter. Le fait d'accompagner un monde en transformation donne au récit un caractère exemplaire.

L'enfance de l'auteur est attendue, nous sommes au XVIIIe siècle, un père francmaçon, bon et révéré, une mère respectée mais qui lui préfère un frère, des parents qui se séparent. Il connaît plus qu'une aisance matérielle mais n'est que de petite noblesse provinciale, il narre ses avatars avec un précepteur, c'est un enfant curieux du monde, sensible, qui lit, il se décrit généreux, d'un naturel attaché à plaire à son père et selon ses dires, ne recherche que les voies de la vertu. Il émaille son récit d'amours enfantines puis adolescentes. Il montre un réel attachement à Dieu, plus pensé que spontané. Fils des Lumières, il n'y a là rien de très singulier. Il décrit par le menu la société de sa province normande, sa vie mondaine parisienne et provinciale, ses goûts littéraires (bien sûr Montesquieu, Voltaire, Rousseau, d'Alembert), s'enthousiasme pour la nature, s'adonne à la pastorale, alors tellement en vogue, admire la mer : «Que l'homme est petit par sa stature, mais qu'il est grand par son génie et son courage » (p. 101).

Trois citations caractérisent l'itinéraire et surtout l'évolution intellectuelle et politique du personnage : «Dès ce moment, les noms des Malesherbes, des La Chalotais ont toujours été sacrés pour moi. Et celui des D'Aiguillon et de ses pareils un objet de mépris. Madame du Barry, les seuls délices de la cour, ses principes immoraux et licencieux m'inspiraient déjà des dégoûts. Mais le roi a toujours été pour moi un être sacré à qui Dieu lui-même avoit imprimé un caractère paternel» (p. 38). Puis, p. 72, à propos de l'admiration qu'il portait dans sa jeunesse à Helvétius : «Plus tard, j'ai reconnu et j'espère avoir réfuté les erreurs funestes dont ce livre est semé. Erreurs qui nous ont fait bien du mal en démoralisant les peuples ». Enfin à propos de Voltaire et Rousseau : "Que Dieu daigne pardonner à ces deux grands hommes, en faveur de leurs bonnes intentions, tout le mal qu'ont produit leurs écrits » (p. 109).

De fait l'homme vieillissant assume les contradictions de la pensée des Lumières, celles de son milieu et de sa classe, il explique ses positions nouvelles mais ne renie pas celles de sa jeunesse, il appelle tout au long de son ouvrage au respect de l'autre, position qu'il nuance, certes, au fil des événements mais le jeune homme de 1778 a sincèrement pensé cela, l'homme des années 1820 rectifie les choses: «D'ailleurs mon caractère s'y opposait, je n'aurais jamais pu supporter d'autre supériorité que celle de la 
vertu, du mérite ou de la place. Quant à la naissance, je croyais valoir qui que ce fût. Je m'accuse aujourd'hui de cet orgueil qui, répandu dans toutes les classes, a été une des principales causes de la Révolution et des maux qu'elle a produits " (p. 130).

Ce jeune nobliau aisé devient militaire, en garnison à Saumur, à Metz, à Lunéville. Ce type de vie en temps de paix, en fait un oisif. Le jeu, les femmes, le duel constituent l'essentiel de ses occupations. Après son retour à la vie civile, il se marie. Désormais ses relations matrimoniales et familiales qu'il rapporte par le menu nous renvoient l'image d'un brave et généreux garçon parfois un peu nigaud. Ses réflexions souvent présomptueuses nous le révèlent candide. Force-t-il le trait?

Dès 1789 , contre l'avis de sa famille et de nombre de ses amis, il accompagne avec intérêt les débuts de la Révolution, il s'inquiète et doute lors de la fuite du roi : "Ce jour-là, il n'y eut pas de populace. Le vrai peuple se montra. C'est le seul jour où je l'ai vue » (p. 519), puis refuse d'émigrer : «Appeler l'étranger pour vous aider à envahir votre pays [...] J'aimerais mieux être pendu ici que triomphant avec vous et avec eux " (p. 520). Il ne cautionne pas l'attitude de sa femme qui assiste à des messes réfractaires, cela l'agace mais il ne peut accepter la phase radicale du processus révolutionnaire. II manifeste alors sa peur des Jacobins, sa haine de Marat, son refus du 20 juin, du 10 août, des massacres de Septembre, redoute les Cordeliers, dénonce Robespierre, Billaud-Varenne, Barère et «Paris ce foyer de corruption et de fureur» (p. 564). À cet endroit il a atteint ses limites politiques. Les lignes qui suivent résument sa pensée : «J'étais réellement démocrate et je le suis encore, si l'on donne ce nom à ceux qui pensent que le bien de la société tout entière, que l'avantage du plus grand nombre doit être le but des pensées, des vœux et des actions de l'honnête homme et que les classes supérieures n'existent que comme moyen de parvenir à ce but. Mais si l'on appelle ainsi ceux qui veulent que le peuple se gouverne par lui-même, je ne l'ai jamais été, je ne le serai jamais » (p. 440).

Sans adhérer à la politique girondine, il précise : « sans approuver leur système, on doit les admirer et gémir sur leur perte" (p. 568). Après avoir refusé d'émigrer une nouvelle fois, par crainte de compromettre femme et enfants, le 13 brumaire an II, il est conduit en prison. Sauvé par le 9 thermidor, il sort le 7 brumaire an III, il a vécu cette année, dans une prison dorée, entre mondanités et peurs. Après sa libération, il se retourne progressivement vers Dieu.

Après 1794, nous le retrouvons dans la peau d'un proto-notable, sa vie quotidienne privée et publique le conduit vers la notabilité telle qu'elle se met en place en France au XIX ${ }^{c}$ siècle. Ses fonctions : en l'an III, président du canton d'Argueil, en l'an IV électeur à Rouen pour l'Assemblée électorale et ce, tout au long du Directoire, même en l'an VI malgré la défiance manifestée contre les nobles après Fructidor. Il rencontre Lemonnier, le fabuliste, l'abbé Sicard, Thiessé, sa conversion religieuse est achevée en 1797-1798. Il fréquente la bonne société rouennaise, constate «le triste état de la République » (p. 738), assiste à Brumaire, résultat de «la ruse de l'abbé Sieyès, l'audace de Bonaparte, l'habileté de Lucien » (p. 739), devient conseiller de sous-préfecture, participe au Conseil d'arrondissement de Neufchâtel, est nommé notable national tel que le définit la Constitution de l'an VIII et président de canton en l'an X.

De par ses bonnes actions (il fait du bien!), grâce à la liste de ses fréquentations, aux rapports qu'il entretient avec ceux qui l'entourent, aux protections ou aux recommandations dont il bénéficie ou qu'il accorde, c'est de l'intérieur que l'on découvre une mise en place de la notabilité et parallèlement l'utilisation de cette influence locale par le régime. Puis très vite il s'interroge à propos de Bonaparte : «Doria ou Cromwell ? ». En 1802, lors de la visite du Consul à Forges, le portrait qu'il dresse de Bonaparte est peu flatteur (p. 764). Après la mort du duc d'Enghien, il se détache progressivement du Premier Consul et totalement après le couronnement impérial. 
Il se dit ravi d'échapper à une sous-préfecture qui est donnée à Albitte, accepte un commandement de cohorte, puis sa notabilité est confirmée par son agrégation à l'Académie de Rouen en 1810. Il achète un remplaçant pour son fils après avoir condamné la Guerre d'Espagne qu'il juge «la plus immorale de toutes celles que nous rappelle l'histoire» (p. 807). Il approuve la "généreuse abdication de Bonaparte» (p. 829), pendant cette année « douloureuse » où il perd sa fille préférée.

Au cours des Cent-Jours, Bonardi s'inquiète d'une vive agitation pro-Napoléon à Rouen. En 1815, il défend publiquement et courageusement le régicide Pocholle, confirmant ainsi son sens de l'amitié et décide de recevoir les Prussiens sur ses terres pour éviter des tracas à «ses paysans ». De plus en plus bigot, royaliste convaincu qui se dit "modéré ", il n'évoque la période 1816-1826 que très succinctement : il continue de distribuer des secours, devient juré en 1819 et condamne l'assassinat du duc de Berry. Il achève la rédaction de ses Mémoires le 24 janvier 1826. Lui qui s'était fait inoculé par Sutton à la fin du XVIIIe siècle, lorsqu'il contracte le choléra en 1832 à Neufchâtel, il refuse l'assistance d'un médecin et meurt le 25 juin 1832.

En rédigeant cet ouvrage, les événements de cette période ne servant que de toile de fond à sa mémoire, il rappelle maintes fois que son but est de mettre et de conserver ses enfants sur le chemin de la vertu. De fait il ne confère de réelle importance qu'à sa vie familiale et domestique. C'est cette volonté de se raconter en distance d'un monde en transformation, qu'il ne fait qu'accompagner, qui donne épaisseur et originalité à ses Mémoires.

Il s'agit donc d'un travail riche et révélateur qu'il serait intéressant de mettre en réseau avec d'autres Mémoires, normands en premier lieu mais aussi de provinces diverses. Enfin il faut saluer la richesse et la précision des notes.

Christine LE BOZEC

Philip T. HoffMan, Gilles Postel-Vinay, Jean-Laurent Rosenthal, Des marchés sans prix. Une économie politique du crédit à Paris, 1660-1870, Paris, Éditions de l'École des hautes études en Sciences sociales, 2001, 446 p.

Comment fonctionne le crédit privé à Paris, de la mi-Xvile siècle à la mi$\mathrm{XIX}^{\mathrm{e}}$ siècle ? La réponse passe très largement par l'analyse des pratiques d'une profession-charnière, celle des notaires. Philip Hoffman, Gilles Postel-Vinay et Jean-Laurent Rosenthal ont donc décidé d'ausculter en longue période les actes notariés, sous toutes leurs formes, qui concernent le crédit privé, sans négliger d'évoquer en contrepoint la place occupée par les emprunts publics et la concurrence au moins potentielle d'autres intermédiaires, banquiers ou marchands. Pour tester leurs hypothèses, il leur fallait évidemment une base statistique suffisante. Bien des chercheurs, depuis les travaux pionniers de Madeleine Jurgens, d'Adeline Daumard et de François Furet, sont allés puiser dans le Minutier central des notaires de Paris le matériau de l'histoire d'une vie, d'une profession ou d'un quartier. Grâce à quelques dépouillements systématiques mais limités - pour la période révolutionnaire ou pour la Restauration - on pouvait se risquer aussi à étendre la prise. L'informatisation des actes des années 1751 et 1761 a changé radicalement la donne en procurant les moyens du quantitatif sériel. Les auteurs des Marchés sans prix.. devaient franchir un pas supplémentaire. Ils ont choisi de suivre mensuellement, de 1660 à 1869, l'activité de crédit d'un échantillon de dix études dont les répertoires ne souffrent (presque) pas de lacune, en en ajoutant trente autres, examinées à des coupes décennales. Au total, un tiers environ des études a été pris en compte. C'est aussi un tiers de la production du notariat parisien, s'agissant encore une fois de la seule fonction de crédit. Les dépouillements de 1751 et de 1761 valident en effet la représentativité des études de référence.

Selon les résultats de cette enquête ambitieuse et minutieuse, les notaires ont joué un rôle majeur dans l'organisation du crédit parce que (et tant que) ils ont disposé d'un 
capital précieux et mal distribué : l'information. En effet, ils étaient, chacun pour leur clientèle, en possession de connaissances précises sur les moyens d'éventuels prêteurs et emprunteurs, ne serait-ce que par le biais des contrats de mariage, de partage et de succession. Collectivement, d'autre part, cette grosse centaine d'intermédiaires (pour une population qui avoisinait 600000 habitants en 1789) tenait à peu près tous les fils, à condition que la concurrence n'étouffe pas l'échange de renseignements ou de clients. L'asymétrie d'informations, inévitable dans une grande cité, a été leur chance. Dès que l'on quittait le cercle rassurant mais limité de la famille ou des proches, comment échapper à ce professionnel pour prêter ou emprunter, fût-ce avec un gage suffisant ? Lui seul saurait appareiller créancier et débiteur, en limitant les risques pour tout le monde. De quels instruments disposent les parties? La rente est la mère de tous les crédits. L'État et les particuliers y ont communément recours. L'obligation, beaucoup moins répandue encore au XVIIe siècle, s'affirme peu à peu, par la souplesse de son emploi. On le sait, l'intérêt n'est pas libre, jusqu'à la Révolution. Il est fixé au «denier vingt " soit $5 \%$. La rente perpétuelle est considérée comme un abandon de capital puisque le prêteur ne peut en exiger le remboursement. Celui-ci dépend du libre vouloir de l'emprunteur. Le versement régulier d'une somme, en échange, est par là moralement justifié. La rente viagère introduit un pronostic ou, pour l'État, de la statistique mais elle repose sur le même principe. Quant à l'obligation, l'échéance de son remboursement a été décidée par le prêteur. Il suffit de stipuler que l'argent rendu sera supérieur à l'argent avancé pour camoufler le versement, à terme, d'un intérêt. Les auteurs décrivent finement, dans la durée, le jeu de la rente et de l'obligation - qui recouvre à peu près la relation long terme/court terme - du point de vue des contractants et aussi de l'État emprunteur. Ce dernier ne provoquerait aucun «effet d'éviction» à l'encontre du crédit privé. "L'appétit d'emprunt gargantuesque de Louis XIV " écrivent Philip Hoffman et ses collègues "ne parvient pas à déprimer le marché du crédit privé ». Avant l'ère révolutionnaire des emprunts forcés et des confiscations, il ne serait surtout question de consolider les dettes publiques, en les amputant au passage. Que ces deux types de crédit soient à ce point déconnectés intrigue mais la démonstration, répétée dans le livre, entraîne la conviction.

Les auteurs distinguent cinq périodes dans la séquence étudiée, du début du règne personnel de Louis XIV à la chute de Second Empire. Sous le Roi-Soleil, le crédit privé est encore largement un accord entre personnes de connaissance, authentifié par un notaire. Il stagne puis recule à Paris, victime de trop d'incertitudes. Les fluctuations violentes des prix de base, les guerres et les dettes publiques, tout pousse à temporiser. Deuxième temps, la crise de Law s'est soldée par une liquidation très défavorable à ceux qui avaient prêté à la Couronne (ils y laissent la moitié du capital engagé), un peu moins pour les prêteurs à particuliers : leur perte est de l'ordre du tiers. Les auteurs pointent deux conséquences de cette aventure. D'abord, le notariat avait tout à perdre, pour ses activités de crédit, à la réussite de la Banque. Il est peu touché par son effondrement et gagne durablement à son discrédit. Du côté des particuliers, ils acquièrent dans l'épreuve une culture de l'anticipation. Aux deux bouts de la chaîne du crédit, on apprend à veiller à la politique monétaire et financière du pouvoir, pour être en mesure de prêter ou d'exiger un remboursement, pour les uns, d'emprunter ou de faire attendre un créancier pour les autres. En cas de crise, qui trouve forcément sa solution, la question ouverte est de savoir à quelles conditions s'effectuera la banqueroute. Autant dire que prêteurs et emprunteurs avisés ont intérêt à solliciter le notaire non pas simplement en tant que greffier, mais comme un courtier expert. Vient, de 1726 à 1789, la période faste du notariat parisien en charge du crédit. Le niveau d'encours atteint à la veille de la Révolution ne se retrouvera à peine que quatre-vingts ans plus tard, alors que la population a presque doublé dans l'intervalle. La Révolution entraîne une cassure nette qui, dans ce domaine, ne sera pas compensée ultérieurement. Face à l'inflation et aux désordres monétaires, tous les citoyens sont actifs, disent joliment les auteurs, signalant par là que l'anticipation devient une attitude de tous les instants. Ils montrent que les 
Parisiens perdent confiance dans la nouvelle monnaie dès avant la fin de 1791 , alors que le gage foncier est encore supérieur aux émissions. Deuxième découverte dans le quotidien des prêts et des remboursements, il semblerait que la Terreur n'ait pas limité ses effets à la contrainte immédiate mais ait ouvert un espoir de vraie stabilisation. Troisième décalage chronologique, l'anticipation sur le retour à la monnaie métallique a conduit à des remboursements massifs dès 1795 , soit, du point de vue des débiteurs, deux ans trop tôt. Dernière phase, une reprise molle. Le crédit organisé par les notaires se trouve concurrencé par les maisons de banque et par le Crédit Foncier qui triomphe dans le prêt hypothécaire. Les tentatives de certaines études de pratiquer le dépôt aboutissent à quelques faillites dissuasives. D'ailleurs la puissance publique y met le holà. De toute façon, l'information sur l'état des garanties immobilières qui faisait la force du notariat au temps du renseignement rare n'est plus son privilège. L'accès à la conservation des hypothèques se banalise au XIXe siècle. Le repli en bon ordre du notariat parisien hors de la sphère du crédit n'implique pas que les études de province suivent le mouvement. Faiblesse du système bancaire et société d'inter-connaissance font durer hors de grandes villes les pratiques anciennes.

Il faut revenir sur les principaux apports de ce livre et sur les questions qu'il fait surgir, pour la période clé qui sépare les deux banqueroutes, celle de 1726 et celle de 1797. D'abord sur la taille et la composition du marché du crédit notarié. La chance des études parisiennes tient à la masse de leur clientèle qui offre une grande variété de situations et d'attentes. Les notaires peuvent donc proposer des solutions adaptées, d'autant plus qu'ils savent profiter, en cas de besoin, des flux inter-études en croisant les clientèles. $\mathrm{Ce}$ marché n'est pas uniquement parisien. Tout dépouillement un peu continu d'actes notariés fait apparaître, au XVIII siècle, des contractants de province ou de l'étranger, le plus souvent représentés par procuration lors de la signature. Le privilège de juridiction, pourrait-on dire, qui permet aux notaires parisiens de ne pas être limités dans un ressort, joue à plein. Il s'agit de faciliter l'accès aux rentes sur la Couronne, sur l'Hôtel de Ville, les États provinciaux, etc. mais aussi de faire entrer ces horsains dans les mécanismes de cette superbe pompe à crédit privé, plus aspirante que refoulante, semble-t-il, qu'est Paris. À cet endroit, on mesure mal comment ces notaires, dont l'information fait toute la puissance, procèdent pour obtenir des renseignements fiables sur cette clientèle, au moins pour le premier contrat. Ils doivent accepter une marge d'incertitude et, partant, de risque. Deuxième caractéristique originale de ce marché, le rôle des femmes. Philip Hoffman et ses collègues insistent sur leur participation massive, sous la forme, principalement, de placements en rentes viagères. Ils observent que les femmes sont d'autant plus présentes que les notaires ne se contentent plus, au XVIII e siècle, du rôle de greffiers pour devenir des conseillers. Or, à statut social égal, les femmes ont beaucoup moins de relations dans le monde, de connaissances, et ont besoin d'être aidées dans leurs démarches financières. L'asymétrie d'information les touche plus que les hommes. Autre trait de cette population, celui-ci plus attendu, sa répartition par âge : les jeunes empruntent pour s'établir - maison, office, etc. - les vieux prêtent pour s'assurer un revenu supplémentaire. À plusieurs reprises, les auteurs indiquent que les contractants appartiennent au même monde, celui des gens aisés. On ne prêterait qu'aux riches. Les artisans et les gagne-petit auraient recours à d'autres formules, dont celle du mont-de-piété. Cette affirmation est heureusement nuancée dans d'autres passages du livre. Il semble bien, en effet, que des personnes issues de milieux modestes aient recours, pour des prêts parfois minuscules, au service des notaires.

L'objectif des Marchés sans prix... est de construire l'histoire - ascension, apogée, déclin - des notaires parisiens comme intermédiaires de crédit. On comprend donc que les autres solutions soient peu présentes dans ce livre. Pourtant, elles ne cessent pas d'être efficaces. Il faudrait éviter, pour réhabiliter le notariat, de tordre le bâton dans l'autre sens. L'appel à la parentèle reste très courant aux XVIII' et XIXe siècle. On le voit, par exemple, quand une société commerciale se constitue. La famille se présente 
comme le prêteur du premier recours. Au-delà de ce premier cercle, il est fréquent de trouver des alliés, des collègues, voire des camarades d'études dans les signataires d'un contrat de crédit. Les maîtres ès-information que sont les notaires n'égaleront jamais la connaissance intime et la confiance vérifiée que chacun peut avoir de ses proches. Les marchands, de leur côté, complètent, plus qu'il ne concurrencent, l'activité de crédit des notaires. Leur soutien s'exerce dans le domaine du très court terme. Des payements à 90 jours, qui sont de règle entre professionnels, aident les trésoreries. On le voit, $a$ contrario, pendant la Révolution, où ces délais disparaissent. Disons plutôt que le crédit change alors de sens : le client - par exemple un maître de forges vis-à-vis d'un marchand de bois - doit au moins pour une première transaction, payer une partie de la facture d'avance. Ensuite, il payera comptant. À l'égard de la clientèle non professionnelle, les marchands sont régulièrement mis dans la nécessité d'ouvrir un crédit sur gage ou sur la réputation de leur débiteur. Le menu peuple vit de ces pratiques. Les Grands, aussi. Natacha Coquery a montré l'ampleur et la banalité de ce crédit que l'aristocratie parisienne, en ses hôtels, extorque à ses fournisseurs. Concernant le système bancaire, il est difficile de suivre les auteurs lorsqu'ils considèrent que le notariat aurait pu aussi bien assurer les investissements lourds dont les maisons de banque se sont chargées. Quelques opérations réussies ne font pas un métier ni ne démontrent la capacité à réunir périodiquement de fonds considérables. Pour financer Anzin, il a fallu Périer. Dans les années 1820 , le boom immobilier dans Paris, la mise sur pied de grandes compagnies métallurgiques et minières, la relance de la construction de canaux ne pouvaient trouver leur financement chez les notaires, à moins que ceux-ci ne se soient syndiqués. La banque répondait mieux à ces nouveaux besoins.

Sur la crise révolutionnaire enfin, les auteurs montrent bien le traumatisme qu'elle a provoqué dans le système du crédit privé. On pourrait y ajouter que le notariat parisien a particulièrement souffert dans cette période - émigration, emprisonnement, guillotine - et que ses réseaux traditionnels d'information n'étant pas mieux traités, la solution de continuité qui l'affecte apparaît encore plus évidente. Un point aurait peutêtre mérité plus de développement, même s'il sortait de l'axe du livre. Les prêteurs qui doivent accepter les remboursements au pair, sans pouvoir les différer, ne sont lésés que s'ils ne réagissent pas. L'achat des biens nationaux fournit le débouché à tout ce papier dévalué ; les remboursements, d'autre part, deviennent une aubaine. De même, dans les transactions ordinaires, ceux qui détiennent des masses d'assignats puis des mandats inemployés peuvent exiger - avec un succès mitigé, il est vrai - de payer des services, des marchandises ou des biens immobiliers en monnaie révolutionnaire. Il n'était pas nécessaire de convoquer les «bandes noires ». Bien d'honnêtes Parisiens ont su - grâce à leur notaire? - profiter des malheurs du temps.

Denis WORONOFF

\section{Steven L. KAPLAN, La fin des corporations, Paris, Fayard, 2001, 740 p.}

Depuis vingt ans Steven Kaplan s'est imposé comme l'un des meilleurs historiens du monde du travail en France à la fin du XVIIIe siècle, en même temps qu'il a publié des ouvrages imposants consacrés à la commémoration du bicentenaire de la Révolution et à la boulangerie actuelle. Ce livre est lui aussi d'un volume considérable, 740 pages dont plus d'une centaine de pages de notes qui sont souvent en elles-mêmes des éléments importants dans la démonstration. Il peut apparaître autant comme une somme sur la fin des corporations qu'une proposition nouvelle d'analyse du mouvement sans-culotte, mais aussi comme une réflexion sur l'historiographie française et anglosaxonne consacrée à la Révolution française, ou encore comme une contribution volontairement impertinente dans le débat contemporain sur le libéralisme et l'étatisme. Avec une alacrité bien connue, soutenue par un style qui recourt parfois à des métaphores ou à des digressions provocantes (comme le titre du chapitre XII : «Encore un mois d'août fatal : la route accidentée vers l'abolition »), l'ouvrage commence par le 
rappel de ce qui peut être un "paradoxe français ", la dénonciation récurrente de tout ce qui peut passer pour une corporation, alors que les impasses du libéralisme comme de l'étatisme suscitent des interrogations qui conduisent à des réinventions néo-corporatistes, si bien que l'épisode corporatiste de l'État français ne peut pas apparaître comme totalement étranger à l'histoire nationale. Le but avoué de l'entreprise est donc de brosser le cadre de pensée qui a vu la disparition officielle des corporations en France, au printemps 1791, sous l'effet conjoint des lois Allarde et Le Chapelier, sans tomber dans les a priori qui sont ordinairement requis pour les comprendre mais au contraire en exposant la complexité et l'ambiguité des mouvements qui ont conduit les Français à ce choix. Pour cela, le livre s'attache à retracer l'histoire qui s'est déroulée de 1776 à 1791 - avec des incursions en amont et en aval - de Turgot à Le Chapelier, prenant au passage l'histoire de la Révolution en écharpe; on en verra les conséquences plus loin.

La trame du livre peut être résumée facilement, puisque la chronologie est respectée et l'auteur a pris le soin de donner en tête de chaque chapitre un résumé, la conclusion fournissant un ultime rappel de l'ensemble. L'histoire commence donc au moment où le ministère Turgot, prolongeant des critiques antérieures, entend supprimer les corporations, les rationaliser et les réorganiser, pour les assujetir administrativement d'un côté et ouvrir le marché du travail à la loi de l'offre et de la demande de l'autre, insistant sur la nécessité de la liberté. La démarche est l'aboutissement d'un double processus, la critique de l'administration royale d'un ensemble peu ordonné et la mise en cause par les Lumières de ce qui apparaît comme un anachronisme. Les physiocrates ont gagné, d'autant plus que les concours d'académies ont préparé l'opinion à la décision. La conviction est partagée que le contrôle de l'État est inutile, car l'intérêt même du marchand ou du fabricant le pousse à être de bonne foi - position dont on voit bien les résonances politiques ultérieures. La difficulté naît pourtant de la position ambiguë de l'État, qui a taxé jusque-là les corporations et qui garantit leur endettement. Les mesures économiques sont donc inséparables des mesures fiscales et induisent aussi des mesures politiques et même morales. Si la démarche du ministère est logique, parce que nombre d'administrateurs sont acquis aux Lumières, elle est fragile car des oppositions sont tenaces entre interventionnistes et libéraux, entre lesquels des réformateurs tentent de trouver des compromis, et même risquée car cela rappelle des souvenirs fâcheux datant de 1716 ou de 1771, quand l'État avait entrepris de contrôler, voire de rénover, les corporations. Si bien que l'image même de l'État est trouble, un réel libéralisme peut côtoyer le vieux fonds colbertiste d'une administration interventionniste et fiscalement toujours avide. Le camp adverse n'est pas plus unifié, sous le vernis unitaire des corporations se cachent des disparités entre les corps, entre les villes, si bien que des volontés réformatrices ne sont pas absentes, même si les identités profondes ne doivent pas être remises en cause. Enfin, les parlements jouent leur rôle dans cette pièce, défendant l'indépendance des corps contre les empiètements royaux, mais s'affirmant clairement dirigistes pour faire face aux disettes. Une conclusion essentielle à cette étape est que les critiques exprimées dans les années 1770 vis-à-vis des corporations ne sont certes pas inédites, mais qu'elles sont dorénavant portées par l'air du temps d'une part et qu'elles sont soutenues par le roi et par (une partie de) l'administration. Le roi est donc modernisateur, il convient même de parler d'un « libéralisme royal » (p. 74) mais qui est dans la nécessité d'imposer ses décisions dans un premier temps. Ainsi chaque catégorie d'acteurs exprime-t-elle des aspirations contradictoires.

Dans ce jeu complexe, Turgot introduit en février 1776 des mesures qui suppriment les corporations mais qui renforcent les inégalités économiques, elles sont lues par les tenants des corporations comme destructrices de l'ordre global, tandis que les compagnons les tiennent pour la possibilité d'obtenir une liberté plus grande. Il s'agit d'une véritable inversion des valeurs sociales, d'une «carnavalisation » de la société, justifiant l'insubordination des compagnons, même introduisant un renversement généralisé des valeurs sociales comme le craignent les parlementaires qui défendent les corps 
intermédiaires comme base indispensable à l'équilibre social. Ces mesures sont pourtant très inégalement appliquées, voire pas du tout dans de nombreuses provinces où les parlements, voire les administrateurs locaux, font une résistance efficace, si bien qu'elles sont abandonnées en mai 1776, ce qui entraîne des bouleversements véritables; car il ne s'agit pas d'un retour à la situation antérieure, mais au contraire de la création d'une situation nouvelle. Le rétablissement des corporations qui n'ont pas été supprimées s'accompagne d'une redéfinition générale des corporations, de leurs rapports entre elles comme avec l'État, puisqu'il ne s'agit rien de moins qu'une véritable refondation des corporations pour tenir compte des relations réelles entre les corps de métier, sur fond de reprise en main des ouvriers; Turgot a bien provoqué un "cataclysme». Un des exemples est donné par la place accordée aux femmes dans le nouvel ordre, tandis qu'elle est toujours refusée aux Juifs. En même temps, les ouvriers déçus par le retrait de Turgot persistent dans leur fronde, enfin les critiques contre le libéralisme se font plus vives, dénonçant un danger réel pour la société.

En tout état de cause, l'opération profite inégalement aux maîtres, car selon les corps et les provinces, la refondation des corporations s'accomplit inégalement, tenant compte des résistances antérieures, de l'inexistence aussi parfois des corporations en tant que telles. En outre ces refondations introduisent l'agrégation à côté de la maîtrise, créant un nouveau statut flou dans la communauté des maîtres. En résultent une fronde continue des ouvriers et des compagnons, une division parmi les maitres dans chaque corporation, comme entre les corporations, puisqu'une partie est reconduite tandis que d'autres sont confrontées à des amalgames qu'elles refusent, enfin une crise de recrutement des maîtres et la confirmation pour beaucoup qu'il est possible de s'établir hors du contrôle corporatif.

Même s'il est difficile de connaître l'état exact des luttes sociales, il est assuré que les années 1780 voient la montée de l'emprise des idées des Lumières, de la volonté réformatrice de l'État, mais aussi des difficultés économiques, le tout entraînant une augmentation des tensions sociales. Le processus n'est ni linéaire, ni simple, mais combiné, le résultat est complexe, mais débouche pour partie sur la montée de l'individualisme, tant patronal qu'ouvrier, sur fond de critiques de la part des corporations reconstituées contre les «faux ouvriers » qui s'installent en toute impunité. Pendant ce temps, le faubourg Saint-Antoine défend bec et ongle sa liberté d'entreprise, hors corporation, et son image d'excellence contre les accusations des chefs des grands corps.

Dans ce climat troublé, la convocation des États généraux introduit un nouvel élément pertubant, puisque les corporations ne sont pas représentées comme elles le souhaiteraient, surtout à Paris, où elles ne trouvent pas de place dans la représentation. À ce qui apparaît comme une injustice s'ajoute la contagion introduite par le nouvel état d'esprit, puisque une partie des maîtres se voit comme le tiers état soumis et résistant aux privilégiés, la formulation est évidemment reprise à leur compte par les compagnons, mais contre leurs patrons. Ce glissement de sens des mots amène les corporations à se proclamer du côté de la Révolution en marche, tout en faisant appel à une réglementation intelligente de la part de l'État. Corporatif et révolutionnaire tentent de se conjuguer, d'autant plus que les ébranlements de la nuit du 4 août et de la Déclaration des droits de l'homme posent la question de la survie des corporations. Mises à l'écart du débat dans un premier temps, alors que certains ouvriers estiment que dorénavant elles n'ont plus d'existence possible, elles subissent ce nouveau traumatisme de façon complexe. Les maîtres dénoncent, évidemment, le chaos, mais ils se posent aussi en révolutionnaires, luttant eux aussi contre les «aristocrates » et se posent en garants de l'ordre social que les demandes ouvrières commencent à mettre à mal. La corporation est nuisible en tant qu'ordre intermédiaire, elle garde cependant une utilité en garantissant la qualité des ouvrages et en encadrant les compagnons. Sur ces deux points, les municipalités, dont celle de Paris, se retrouvent du côté des maîtres dès 1790 , et font face aux associations ouvrières contestatrices qui naissent à cette époque. 
Dans cette difficile conciliation des principes révolutionnaires et de la vie quotidienne, la question des corporations devient une des pierres de touche des choix politiques, car le langage politique est interprété librement par toutes les parties, qui se réclament de leur nouveau statut de citoyen et ne veulent pas se retrouver assimilées à la Contre-Révolution. Dans les luttes sociales de nouvelles qualifications sont usitées, si bien que les ouvriers sont dénoncés comme des «brigands", tandis que les maîtres deviennent des «monstres aristocratiques ». Mais la crainte de la subversion ouvrière est primordiale devant la naissance de nouvelles formes associatives qui inquiètent maîtres, administrateurs et juges de paix. L'exemple est donné par l'Union fraternelle des charpentiers qui se constitue mi-amicale, mi-syndicat, et qui se retrouve, par force, dans la mouvance du courant cordelier. Les maitres de leur côté créent les structures clés de la sans-culotterie. Dans ces échanges, les positions sont souvent contradictoires, les modérés sont plutôt libéraux économiquement, mais n'entendent pas céder aux pressions ouvrières; les courants plus extrémistes qui ont peur d'une dissolution du lien social par le pouvoir de l'argent, réclament l'interventionnisme de l'État soit pour protéger les ouvriers, soit pour empêcher les troubles. La question est tranchée par le décret Allarde qui, dans une optique essentiellement fiscale, supprime les corporations et crée la patente comme moyen d'accession au patronat, puis par la loi Le Chapelier, qui interdit toute association dans une optique répressive contre les menaces de subversions. S'agit-il comme le dit Kaplan d'une mesure d'inspiration révolutionnaire qui règle un problème d'Ancien Régime ou comme l'affirment des critiques royalistes d'une mesure imitant l'Ancien Régime et traitant un problème né de la Révolution? La question reste ouverte, sauf à dire que Le Chapelier n'est pas le visionnaire porteur des aspirations de la bourgeoisie industrielle du XIXe siècle, mais bien le juriste, porté par un courant d'opinion ancien, qui établit le contrôle social réclamé par la sanior pars.

Les questions liées au travail ne sont plus ainsi traitées spécifiquement, mais dans un cadre politique, ce qui explique que patrons et ouvriers s'engagent dans l'univers politique, nommément dans la sans-culotterie, qui est l'objet d'un chapitre ultime dans lequel l'auteur propose d'y voir le lieu où les patrons exercent leur contrôle sous couvert d'unanimisme patriotique. L'histoire agitée des corporations dans la vie française est l'occasion de montrer comment les arguments et les positions peuvent s'échanger au fil des luttes sociales et politiques et trouver des résonances inattendues. Le livre se veut clairement de ce point de vue une leçon de méthode. À de nombreuses reprises, l'auteur s'engage dans des discussions historiographiques, le plus souvent menées dans l'épaisseur des notes mises en fin d'ouvrage, mais le dernier chapitre est pour l'essentiel consacré à la discussion des thèses de Soboul, Burstin, Sonenscher et Sewell sur la formation de la sans-culotterie. Ce qui est défendu ici est d'une part qu'il n'y a pas à chercher d'explications linéaires et simplistes, mais plutôt à comprendre des processus contradictoires qui débouchent sur des formations imprévues. La naissance de la sans-culotterie est pour l'auteur l'exemple de ce précipité chimique imprévisible qui, sous l'effet des nouvelles conditions provoquées par la Révolution, naît de la rencontre entre des attentes et des courants anciens, métamorphosés par l'événement. Il est possible de discuter dans le détail de la compréhension de la sans-culotterie, reste que la démarche est convaincante, liant étroitement structure et événement, ne se perdant pas dans des recherches généalogiques illusoires, dépassant ce qui est posé comme contradictions, insistant sur les mutations de sens des mots et des concepts, récusant les jugements trop rapides. Il rappelle notamment qu'il n'y a pas lieu de s'étonner qu'il y ait eu accord sur la loi Le Chapelier, dans la mesure où les données du débat étaient bien connues depuis des décennies, la loi étant de ce point de vue un aboutissement attendu. De la même façon, qu'il ait fallu attendre 1791 pour la voir promulguer n'est pas non plus un sujet d'étonnement, dans la mesure où les urgences étaient plus grandes dans d'autres domaines.

Deuxième leçon du livre, l'auteur attire à maintes occasions l'attention, sur la délicate articulation entre discours et "réalité " et la compréhension des représentations 
des acteurs de l'histoire. Il n'y a pas lieu de prendre au pied de la lettre les discours des corporations ou des ouvriers à aucun moment de l'histoire, il est même nécessaire d'introduire un "coefficient de dédramatisation " sur toutes les réclamations, pour tenir compte de la complexité du réel, de la désunion de chaque camp, de la part d'invention qui se trouve dans ces discours. La discussion sur la nature de la sans-culotterie est au cœur de cette réflexion, puisque l'auteur conteste les analyses qui prennent sans précaution les proclamations sans tenir compte suffisamment des structures sociales sousjacentes cachées précisément par ces allégations. Il est possible de le suivre dans cette voie qui ne prend pas au pied de la lettre les dénonciations de l'absolutisme royal, puisque l'on voit les agents du roi engagés dans des négociations avec leurs partenaires, mais à l'intérieur même de l'administration, comme il ne faut pas croire les déclarations des tenants des corporations, d'autant plus fortes qu'elles sont données au moment d'une refondation qui doit faire oublier les tares antérieures. Plus profondément encore, l'analyse est stimulante dans ce qui est dit de la nature véritable du lien corporatif, dont l'efficacité est moins économique que sociale. Le discours tenu se situait bien au niveau économique, mais la cohésion cherchée était idéologique plus qu'économique, car de tous temps les écarts à la règle étaient considérables et bien connus. En revanche, l'atteinte aux normes admises entraînaient ipso facto un renversement des valeurs, qui allait toucher toute l'épaisseur de la société au-delà des seules structures économiques corporatives. C'est en cela que l'épisode traumatique de 1786 est créateur d'une nouvelle donne sociale et politique, engageant le pays dans une désacralisation et une décorporéification de la société, annonçant l'atomisation des liens sociaux provoqués par la Révolution. Là encore, le livre apporte des éléments de réflexion en montrant bien comment ces idées qui travaillent profondément le tissu social ne sont pas véritablement opératoires avant que les événements factuels des 14 juillet et 4 août 1789 n'aient clairement changé les choses. Comme dans une réaction chimique, les éléments dispersés se précipitent et créent de nouvelles formes. De ce point de vue, l'auteur donne certes raison à ceux qui voient le début de la Révolution bien avant 1789 , la «matrice de la Révolution " est présente dès 1786, mais il renforce surtout ceux qui insistent sur la cristallisation événementielle, indispensable à la mutation des esprits. De ruptures en cristallisation, de malentendus en fixations idéologiques inédites, le livre explore ainsi les ruses de la raison économique et politique qui ont traversé les tactiques des maîtres, des compagnons, des administrateurs et des parlementaires, se situant bien au-delà du champ apparemment limité d'une étude du monde du travail ou d'une réflexion sur la « fin des corporations», comme le titre pourrait le laisser croire. C'est tout le processus de la Révolution française qui est ainsi éclairé au travers d'un domaine particulier, ce qui fait un des intérêts essentiels du livre.

Il convient enfin d'insister sur l'aspect peut-être le plus paradoxal de l'ouvrage, et qu'il ne faudra pas oublier dans les discussions à venir. D'un bout à l'autre, l'auteur insiste sur l'aspect heuristique de sa démarche, sur sa volonté de proposer une interprétation, et il rappelle avec une insistance parfois inquiétante pour le lecteur attendant des conclusions définitives que, malgré les notes de référence qui donnent lieu à une centaine de pages, il ne s'appuie que sur des dépouillements limités, sur des «miettes d'archives », qu'il faudra donc multiplier les études locales et les analyses fines. Ce qui est en jeu ici n'est pas la description achevée d'un processus, mais bien l'ébauche d'un cadre interprétatif qui reste à démontrer dans les détails. À n'en pas douter, ce livre va relancer de nombreuses discussions tant sur les méthodes de l'histoire sociale, que sur les conditions mêmes de la naissance de la sans-culotterie, il participe en cela au renouvellement et à l'actualisation des problématiques de recherche et mérite d'être lu attentivement.

Jean-Clément MARTIN 
Pascal Brioist, Hervé DrÉvillon et Pierre Serna, Croiser le fer. Violence et culture de l'épée dans la France moderne (Xvie-Xvme siècle), Seyssel, Champ Vallon, $2002,429 \mathrm{p}$. touche !»

«Si tu ne viens pas à Lagardère, Lagardère ira à toi ! », «À la fin de l'envoi, je

L'enfance de longtemps envolée, nous gardons tous en mémoire les répliques des héros dans les romans de cape et d'épée. Et si toute cette littérature «à panache » dissimulait l'appétence morbide de duellistes plus criminels que sauveurs de la veuve, de l'orphelin ou... des ferrets de la reine ? Dans son livre, Le Duel dans la société française des $X V^{e}-X V J^{e}$ siècles, essai de psychologie historique, Billacois «construit » le duel, en présentant l'histoire comme expression d'une éthique nobiliaire rétive à l'étatisation. Les trois auteurs - on allait écrire les trois mousquetaires - ambitionnent de le "déconstruire " en retirant pierre à pierre tous les systèmes bâtis pour le justifier, l'enjoliver ou le transformer en fiction romanesque. Le duel est une perversion, un goût malsain de «roués» qui abaissent l'autre avant de le tuer. Pour le montrer, les trois chercheurs entreprennent d'étudier tour à tour la civilisation de l'épée qui alimente une civilisation de la violence, l'escrime, art de tuer comme art de vivre, la république des duellistes, enfin l'encre et le sang ou comment l'escrime influence et corrompt parfois «l'escrire ».

Réaliser un tel projet, c'est se rendre maître de sources imprimées multiples et variées depuis les traités d'escrime européens qui se comptent par centaines jusqu'aux réflexions morales sur le duel comme celles de l'abbé Grégoire, aux traités de combats singuliers et aux journaux de l'époque révolutionnaire comme Les Révolutions de France et de Brabant ou Les Révolutions de Paris. C'est aussi aller se remplir les poumons de la poussière des Archives nationales, des manuscrits de la Bibliothèque nationale ou de ceux du Service historique de l'Armée de Terre. "Un pour tous, tous pour un!", les trois auteurs n'hésitent pas à le faire et l'érudition à laquelle ils parviennent suscite l'admiration. Les notes infrapaginales qui éclairent, soutiennent, ouvrent enfin les chapitres sur des problèmes nouveaux sont un vrai régal qui s'ajoute à celui procuré par un texte clair et bien argumenté. Le critique scrupuleux s'essouffle à trouver la faute ou la coquille (aus au lieu de aux, p. 415, ligne 12).

Vifs et allègres, les trois auteurs nous attirent et nous retiennent. Avec eux, nous découvrons l'espadon, la forte épée ou la lansquenette, leurs procédés de fabrication et leur maniement sur les champs de bataille ou dans les salles d'escrime. Tuer son homme est une science qui demande un long apprentissage et une pratique continuelle. L'escrime sert avec la danse ou l'équitation à former le gentilhomme, elle apprend surtout «à tuer vite et bien». Dans la république des duellistes, on tue dans le silence : le pouvoir interdit les duels, les juges se taisent devant les combats d'épée qui, de Paris à la province, témoignent d'une France violente. Les cadavres s'amoncellent. Pierre Serna les dénombre et les examine minutieusement. Il recense et analyse les plaies pour « faire parler» les dépouilles. Il y parvient avec une habileté et une science qui laisseront pantois Patricia Cornwell, auteur célèbre de romans policiers.

Le port de l'épée, privilège des nobles et de quelques bourgeois, leur permet de vider querelle avec rapidité. Au siècle de la Raison, comme aux siècles précédents, on s'étripe à qui mieux mieux pour les sujets les plus futiles. Encore sait-on mieux tirer l'épée et mieux parer les coups que jadis. Si l'escrime ne pacifie pas les mœurs, elle accoutume les bretteurs à maîtriser la violence et leur donne un savoir-faire qui leur permet d'éviter la mort ou, s'ils le veulent bien, d'épargner la vie de leurs antagonistes. Davantage de duels, moins de morts! Dans une société où l'affrontement des corps structure encore le social, le duelliste apparaît aux auteurs comme "une figure déformée mais complémentaire et inquiétante de l'Homme des Lumières ».

La littérature moralisante n'empêche pas les assauts meurtriers et les hommes de lettres ressortent contaminés de leur examen du duel. Leurs plumes deviennent épées 
qui griffent le cuir de leurs adversaires. Au temps de la Révolution, les mots employés par les journalistes des deux bords sont comme autant de touches qui, zébrant l'ennemi, le désignent et le conduisent à la mort. Pour certains, l'épée est le dernier et suprême argument. Le XIXe siècle inscrit le duel comme un délit sur les tables de la Loi sans que les combattants prennent toujours conscience de la radicale délinquance de leurs gestes. Et puis voici que la plume sauve l'épée. Les journalistes abandonnent le pré où ils se battaient au petit matin clair pour retrouver leur écritoire. Ils y composent une histoire légendaire qui nettoie les duellistes des macules du crime. Revanche du noble sur la société des notables : il est, l'épée à la main, redresseur de torts, un héros populaire.

Le livre suscitera sans doute des polémiques. Elles témoigneront de la richesse d'une entreprise qui est une histoire totale, «attentive aux objets, aux gestes, aux pratiques sociales et aux courants intellectuels, de la Renaissance aux Lumières». L'historien de la société militaire en Révolution que je suis regrette que les auteurs n'aient pas consacré un développement à la transformation de l'épée « de l'aristocrate » en «sabre national» dit d'honneur. Donné comme récompense aux «vainqueurs de la Bastille » ou aux soldats de Jemmapes, il porte sur sa lame une inscription qui en fait le vecteur des vertus civiques. Dans l'imaginaire de l'an II, l'arme des duellistes de la société d'Ancien Régime devait en être comme «régénérée ». Critique mineure pour un ouvrage qui... à la fin de l'envoi, touche!

Jean-Paul BERTAUD

David A. BeLl, The Cult of the Nation in France. Inventing Nationalism, 1680-1820, Cambridge (Mas.) et Londres, Harvard University Press, 2001, 304 p.

David Bell, professeur d'histoire à la Johns Hopkins University, ne considère pas la nation comme une réalité dont l'essence aurait été définie par les Français du XVIIIe siècle. Dans la lignée de réflexions anglo-américaines (en particulier les travaux de Benedict Anderson, Ernest Gellner, Éric Hobsbawm et Anthony Smith), il l'appréhende plutôt comme un produit de la volonté politique, par le fait d'un artefact politique à construire dans un processus d'invention du nationalisme. Il donne ainsi une importance toute particulière au moment où les penseurs français, dans les années 1770 et 1780 , commencent à imaginer la nation sous la forme d'une construction politique, et non comme un fait naturel.

Du point de vue méthodologique, cet ouvrage se situe alors à lả croisée de l'histoire des concepts et de l'histoire culturelle. De l'histoire des concepts, issue des travaux fondateurs de John Pocock et Quentin Skinner, David Bell retient la nécessaire analyse de la transformation des concepts fondamentaux du nationalisme en construction, bien sûr nation et patrie, mais aussi société, civilisation, public, etc. Il s'agit alors d'étudier l'évolution de la signification de ces concepts dans les contextes les plus larges et les plus divers possibles. La dimension d'histoire culturelle intervient plutôt dans la mise en évidence des thématiques fondatrices de la nation, et essentiellement le fait religieux.

Le premier chapitre s'intitule en effet The National and the Sacred. Dans la lignée des travaux de Marcel Gauchet (Le désenchantement du monde, 1995), David Bell émet l'hypothèse que le désenchantement du monde, nettement perceptible à la fin du XVIIe siècle, rompt le lien entre l'ordre terrestre et l'ordre céleste tout en maintenant les virtualités transformatrices du christianisme revivifié par le mouvement de la ContreRéforme. C'est à ce titre que la construction de la nation relève d'emblée d'une mystique, et le restera. Ainsi la patrie apparaît à la fois comme «le premier principe social " et une "seconde Divinité ", alors que, sous la plume de Rousseau, la construction de la nation est rapportée au geste fondateur de Moïse qui «forma et exécuta l'étonnante entreprise d'instituer en corps de la nation un essaim de malheureux fugitifs [...] osa faire de cette troupe errante et servile un corps politique, un peuple libre ». Nous ne sommes donc plus dans le cadre de la construction de la Cité, par référence à 
l'humanisme civique. Selon David Bell, l'invention du nationalisme procède ici d'une pratique culturelle inscrite dans la continuité de la réformation catholique.

Le chapitre 4 sur le culte des grands hommes (« National Memory and the Canon of Great Frenchmen »), qui ouvre un débat avec Jean-Claude Bonnet et son étude sur La Naissance du Panthéon. Essai sur le culte des grands hommes (1998), approfondit cette approche de la dynamique nationale dans le rapport au sacré d'une communauté humaine, donc hors de toute considération sur la providence divine. Jean-Claude Bonnet considère que le culte des grands hommes ouvre une brèche symbolique dans la machine de l'absolutisme royal. David Bell montre différemment que ce culte est tout à la gloire du patriotisme royal dont il a mis en évidence l'importance dans le chapitre 2 sur The Politics of Patriotism and National Sentiment : un patriotisme certes basé de façon émotionnelle sur l'amour du roi, et son identification avec l'idée de patrie, donc soumis au risque d'un retournement contre le roi si sa personne n'est plus l'objet de l'affection de ses sujets.

Cependant David Bell montre aussi le lien entre le culte national des grands hommes et le radicalisme révolutionnaire, en partant du contexte plus large du "républicanisme masculin » de la fin du siècle. Il souligne alors que ce nouveau culte républicain critique implicitement la croyance en l'œuvre civilisatrice des hérö̈nes féminines, et par là même le concept de civilisation, proposant ainsi une approche exclusivement masculine de la patrie. Cette relation contradictoire entre l'imaginaire républicain de la nation et le rôle des femmes est précisée dans le chapitre 5 intitulé « National Character and the Republican Imagination ". Alors que le siècle des Lumières prête à "l'esprit national " français les caractères de la sociabilité, de l'honnêteté, de la politesse voire de la légèreté, considérant donc que « la Société ne peut subsister sans la fréquentation des femmes » qui «font les mœurs », la critique républicaine des années 1770 et 1780 s'inquiète d'une telle corruption du caractère des hommes par la frivolité des femmes. Et, pendant le Directoire, Boucher-Laricharderie (De l'influence de la Révolution française sur le caractère national) se réjouit, non sans un certain cynisme, que la fin de la «domination » des femmes soit acquise depuis le 14 juillet 1789 , et que la Révolution renferme les épouses dans l'intérieur des familles!

La démarche de David Bell consiste bien à montrer les étapes de la formation des concepts de nation et de patrie, et leurs contextes respectifs, donc à souligner leur dynamique créatrice de potentialités contestatrices de l'absolutisme royal, sans pour autant poser $a$ priori leur caractère progressiste. Il montre ainsi l'impact, dès le début du siècle, du «style Ancien Régime» des parlementaires parisiens dans l'ouverture d'un débat autour du nécessaire "amour de la patrie » avant même que le tribunal de l'opinion publique intervienne, au milieu du siècle, pour modifier les termes du débat avec son déploiement à l'horizon des notions réflexives de public et de société. Mais il en vient, de façon plus risquée, à prêter, en fin de parcours, au nationalisme républicain français des caractères particulièrement conservateurs, et en premier lieu, comme nous l'avons montré, l'antiféminisme. Un autre chapitre accentue le trait : il s'agit du chapitre 3 intitulé significativement English Barbarians, French Martyrs.

David Bell propose ici une analyse approfondie d'un poème intitulé Jumonville (1759) qui fait référence à l'envoyé français massacré sur la frontière du Canada par des soldats anglais, en prélude à la guerre de Sept ans. En qualifiant l'Anglais, dont le génie embrassait antérieurement l'Univers, de «nouveau barbare», de "peuple parjure », de «peuple assassin », etc. l'auteur, M. Thomas, recentre le rôle universel des nations européennes autour de la France. L'ouvrage de Lesuire sur Les sauvages de l'Europe (1760), inscrit dans le même contexte, accentue une telle différence intra-européenne entre Anglais et Français. David Bell y voit alors les premières manifestations d'un nationalisme à fondement raciste (" a race-based nationalism ", p. 104).

Une telle analyse des dérives nationalistes, et donc réactionnaires, du processus de construction de la nation française fait débat. Dans la conclusion, David Bell ne nie pas, 
après avoir adopté un point de vue fructueux sur la dynamique spécifique de la construction de la nation française, en être venu à montrer un processus non plus de diversification, mais d'homogénéisation au détriment de la diversité nationale, et au profit de l'imaginaire d'une essence nationale. À ce titre, le chapitre 6 intitulé National language and Révolutionary Crucible concentre les problèmes posés par cette démarche originale. Complétant une publication antérieure, David Bell étudie le processus de politisation du langage dans le nouveau cadre national de la Révolution française. Il s'en tient strictement à la question de la politique d'uniformisation linguistique, restriction qui lui permet d'éviter le débat avec les travaux des historiens linguistes sur la construction nationale de "la nouvelle langue politique". Il émet alors l'hypothèse d'une opposition entre les élites et les patriotes locaux, plus précisément entre la solution «protestante » de Grégoire, qui veut imposer le français standard, et la position des républicains locaux favorables à l'usage politique des idiomes non français, dans la lignée du mouvement catholique d'évangélisation mais en réaction à l'attitude contre-révolutionnaire des prêtres réfractaires. Si l'engagement révolutionnaire et ses manifestations langagières ne sont pas directement issus des précédents religieux, ces précédents structurent, selon lui, les termes du débat à l'exemple de l'opposition susdite. David Bell fait ainsi le choix méthodologique, dans le contexte de la recherche anglophone, de l'approche structuraliste de Pocock, qui privilégie la filiation «passéiste » entre une tradition et ses manifestations, au détriment de la démarche plus réflexive et pragmatique de Skinner, qui met l'accent sur ce que fait un acteur/auteur en disant ce qu'il dit. Ce choix présuppose, dans le cas présent, une unité en devenir des caractères du nationalisme français au détriment d'une approche des normes sociales et des conventions rhétoriques mises en place progressivement pour permettre aux hommes des Lumières, puis aux révolutionnaires d'inscrire la nation - de l'individunation, expression de la liberté personnelle, au français national, expression de la langue de la liberté - à l'horizon d'une multiplicité d'expérimentations émancipatrices. C'est ainsi que David Bell en vient à déceler, en fin de parcours de la construction de la nation, les premières manifestations des traits du nationalisme sans l'individu-nation tel qu'il s'impose en France au XIXe siècle.

Soulignons enfin une caractéristique particulièrement originale de cette publication : elle est enrichie par d'importants compléments sur le site internet de l'auteur, en particulier l'intégralité des citations originales en français et quelques textes de référence, par exemple l'étonnant poème Jumonville. La lecture de cet important ouvrage en est ainsi singulièrement enrichie.

\section{Jacques GuILHAUMOU}

Louis BERgÈs, Valmy, le mythe de la République, Toulouse, Privat, 2001, 157 p.

C'est dans la collection «Entre légendes et histoire » que paraît cet ouvrage dont l'auteur est aussi celui d'une fort belle thèse consacrée aux résistances à la conscription dans les départements aquitains du Directoire à la fin de l'Empire. C'est dire que L. Bergès s'est proposé de traiter davantage l'histoire des mythes auxquels Valmy a donné lieu plutôt que l'histoire de la bataille elle-même, et d'analyser le processus politique qui explique leur apparition et leur survivance.

Certes, l'historien ne fait pas l'économie d'un récit du combat et la première partie de son ouvrage s'intitule "Mystères et certitudes autour d'une bataille". S'appuyant sur les travaux antérieurs de nombreux historiens, L. Bergès rappelle le contexte politique de Valmy mais aussi son contexte militaire. Replaçant la bataille dans l'histoire de la guerre au XVIIIe siècle et dans celle du débat stratégique et tactique de la fin du siècle, il souligne la supériorité technique de l'armée de la Révolution et la possibilité que soient appliquées dès lors les idées nouvelles dont Guibert fut le héraut. Il insiste à juste titre sur le fait que, si Valmy ne fut pas une grande bataille à l'instar d'Austerlitz ou même de Jemmapes quelques mois plus tard, le mot de «canonnade " 
employé d'emblée par des témoins pour définir le combat n'est pas péjoratif ni réducteur. Ce duel d'artillerie remarquable par son ampleur même s'il fut peu meurtrier - mais le nombre des blessés par mutilation est beaucoup plus significatif, remarque l'auteur judicieusement - préfigure une guerre nouvelie. Sur le seul plan technique, Valmy est la naissance - assez peu entrevue par les contemporains, voire les historiens du nouvel art militaire que Guibert préconisait : c'est un des points forts de l'ouvrage que de l'avoir mis en lumière.

Au cours du récit circonstancié qu'il donne du combat, l'historien fait la part belle à l'ínattendu qui défia les prévisions des contemporains et qu'il a dénommé « mystères ». Ils n'étaient pas forcément tels mais ils alimentèrent des légendes contradictoires auxquelles il consacre sa seconde partie : " La constitution du mythe ". La résonance de l'événement n'est pas immédiate, elle date du départ des Prussiens, le 29 septembre. Mais dès la prise de conscience de son importance - la Révolution a remporté contre toute attente une victoire défensive au moment où naissait la République - des interprétations divergentes s'affrontent autour du sens qu'il convient de lui accorder. Sous l'impulsion des représentants de la Convention envoyés dans les départements, a lieu la première célébration nationale de Valmy : ce sont les idées et les idéaux de la Révolution qui ont vaincu parce qu'ils étaient bons et pas seulement les combattants. La victoire justifie - a posteriori - la valeur de ces idées. Quant aux soldats de la République, on ne distingue pas parmi eux ceux de la ligne, ceux des bataillons de volontaires voire les populations locales mobilisées; ils sont considérés comme l'avantgarde de la «cité nouvelle». L'auteur aurait pu souligner davantage le fait que, si la Révolution fait de Valmy un événement fondateur, elle n'en fait pas encore un mythe, tout au plus par cette confusion crée-t-elle les éléments qui serviront ultérieurement à la construction du mythe.

Quant à la Contre-Révolution, elle développe presque immédiatement une légende noire. Ses partisans, comme le dit avec raison $L$. Bergès, sont les vrais vaincus de Valmy. Faute de minimiser ce qui est une très lourde défaite pour eux, ils la dévalorisent en la transformant en combat truqué, fruit d'une ou de plusieurs machinations où le complot franc-maçon a la part belle. Thiers a fait justice de cette légende noire dont il a démonté un à un les arguments.

En effet, Valmy a été aussi objet d'étude pour les historiens : assez tôt, dès l'an VI, pour les historiens militaires dont $\mathrm{L}$. Bergès montre avec raison le souci d'analyse rationnelle. S'il y a démythification de leur part, c'est bien celle de l'invincibilité de l'armée prussienne. En revanche, si les historiens libéraux de la Restauration manifestent encore le même souci d'objectivité, c'est aux historiens romantiques de la monarchie de Juillet que la bataille doit sa dimension d'épopée. Michelet notamment est à l'origine d'un aspect du mythe appelé à faire fortune et que l'on retrouve chez Chassin et Quinet sous le Second Empire : Valmy est la victoire d'un peuple spontanément dressé contre l'envahisseur et qui triomphe, sans instruction préalable, en raison de la justesse de sa cause et de sa foi dans les idées de la Révolution.

On regrette d'autant plus que l'auteur ait négligé l'interférence du mythe avec les débats auxquels donnèrent lieu les grandes lois militaires du XIXe siècle et son influence sur la pensée républicaine en matière d'armée nationale. Certes, en 1818, la bataille où l'on tendait à ne voir que des volontaires parmi les combattants, ne pouvait guère être évoquée par les partisans de la loi Gouvion-Saint-Cyr qui, de fait, rétablissait la conscription. C'étaient au contraire les ultras qui prônaient le volontariat... Certes, en 1832, le vote de la loi Soult fut précédé d'un débat moins passionné sur le plan idéologique mais les républicains et les orléanistes de gauche commencèrent à déplorer la caricature d'armée-citoyenne à laquelle aboutissait la conscription des monarchies censitaires, au nom des valeurs de 1789 en général, toutefois la mythification de Valmy n'était pas encore à l'œuvre pour qu'ils puissent l'invoquer précisément. Mais c'est au moment de la discussion de la loi Niel en 1867-1868 que la mystique du peuple 
invincible parce qu'animé par un idéal supérieur, dans laquelle les républicains des années trente et quarante s'étaient gardés de tomber, conduisit les républicains des années soixante aux surenchères que l'on sait, à la revendication de la suppression des armées permanentes et du désarmement.

On suivra davantage l'auteur quand il analyse les leçons que les républicains tirèrent de Valmy après 1870 . L'échec du mythe est patent lors des vaines tentatives de trouée en masse de la garde nationale parisienne et - ajouterions-nous - sur les champs de bataille chez les troupes levées à la hâte par Gambetta. Cet échec fut répercuté par des polémistes dans la lignée de Rousset qui, avant même la fin de la guerre, fustigèrent les volontaires. Il aurait été bon de souligner, en outre, que l'armée quasi professionnelle qu'était devenue l'armée française subit elle aussi une lourde défaite.

Aussi le symbole de la bataille - l'action conjointe et victorieuse des soldats de ligne et des soldats-citoyens - et non plus le mythe, fut utilisé par les républicains de la IIIe République pour préparer l'opinion à l'instauration progressive du service personnel obligatoire imposé à des civils parce qu'ils étaient citoyens mais qui seraient encadrés par des professionnels.

La troisième partie, "Succès du Mythe » est consacrée aux prolongements de celui-ci et à la résurgence du mythe contre-révolutionnaire. Dans un second chapitre qu'on aurait préféré voir figurer dès le début de cette partie, L. Bergès montre la précoce internationalisation de la légende dans sa dimension idéologique de guerre révolutionnaire et populaire qui persista au XIXe siècle en Europe et en Amérique latine notamment quand y surgirent les mouvements nationaux. Valmy fit figure de guerre d'indépendance, de même au $\mathrm{XX}^{\mathrm{e}}$ siècle lorsqu'éclatèrent les mouvements de décolonisation.

En France, après le centenaire au cours duquel la bataille fut célébrée avec une relative discrétion, la légende devint consensuelle en raison du ralliement des catholiques à la République mais aussi du développement d'un nouveau nationalisme. Mais alors le symbole républicain se transforma en symbole national : on ne vit plus dans le combat que la manifestation de l'éternel génie militaire de la France. Encore insistat-on - et certains historiens eux-mêmes - sur le rôle déterminant des troupes de ligne, anciennes troupes de la monarchie. Il n'est pas jusqu'aux républicains qui ne soient gagnés par ce consensus même s'ils maintiennent face à ceux qui dissocient désormais République et nation, Révolution et Patrie, la dimension idéologique à travers le souvenir idéalisé du soldat-citoyen. Ce souvenir idéalisé explique aussi que les « ruptures» des socialistes et des syndicalistes aient été moins fortes qu'on ne le croit. Quant à J. Jaurès, il est dans la lignée des historiens qui, à l'instar de Chuquet avant lui, tentent d'approfondir la connaissance scientifique de l'événement.

Quoi qu'il en soit, cette vision unanimiste de Valmy que l'on retrouve dans les manuels scolaires du début du siècle prépare l'union sacrée dont elle devient une référence essentielle au moment où éclate la guerre de 1914. Mais après la victoire, le consensus vole en éclats, on assiste à une « réidéologisation» de Valmy dont L. Bergès montre qu'elle s'accentue lors du Front Populaire et qu'elle est perceptible dans les livres de classe. La victoire redevient victoire révolutionnaire plutôt que nationale et celle du peuple plutôt que celle d'une armée. Parallèlement à l'invocation de la légende par la Résistance, le mythe contre-révolutionnaire, surtout sous son aspect de complot franc-maçon refait surface sous Vichy. Les divergences se poursuivent sur le sens de la bataille, voire sur son existence, pendant la guerre froide tant ces affrontements sont conditionnés par des clivages idéologiques. Mais mythe et contre-mythe s'effritent dans les années soixante en même temps que le combat perd sa place dans les manuels.

Il est encore objet de débat entre les historiens : n'est-ce pas reconnaître qu'il est en train de devenir sinon un «objet froid», du moins un objet scientifique ? À ce titre, 
nous ne sommes pas persuadé que l'ouvrage de J.-P. Bertaud «vole au secours du mythe national et populaire» comme le prétend l'auteur. D'une part, il est déjà relecture des légendes «dorée » ou "noire ", d'autre part et surtout, il montre le rôle décisif dans la bataille des troupes ci-devant royales mais en réévaluant leur insertion dans le processus révolutionnaire et en dépassant l'opposition simpliste que l'on a établie longtemps entre elles et les volontaires. Ainsi quand $L$. Bergès affirme en conclusion que Valmy représente un chantier ouvert, il semble que les registres de contrôles des troupes qu'il donne comme exemple de champ historiographique à défricher l'ont été largement par la recherche novatrice de l'historien américain S. Scott et de J.-P. Bertaud : les soldats de ligne étaient au même titre que les volontaires des soldats-citoyens.

La quatrième partie, «Réactualisation du Mythe », à la fois dresse un bilan et trace des perspectives d'avenir. Avec beaucoup de finesse, l'historien reprend ce qu'il avait évoqué antérieurement, la transformation de Valmy, en lieu de mémoire qui ne se fit que lentement en fonction des avatars du mythe et ne commença véritablement que sous la monarchie de Juillet. Avec une plume alerte, il évoque l'épisode de la commémoration sur le site à l'automne 1989 qui fut une commémoration-prétexte. Enfin ultime - et paradoxal - avatar de la légende, c'est au moment où disparaît l'armée de conscription (que n'était pas l'armée de Valmy par ses structures mais qu'elle préfigurait par son esprit) que les pouvoirs publics envisagent de placer le 20 septembre, la journée de préparation à la défense. L. Bergès estime que ce choix serait un couronnement, redonnant un sens non au mythe mais à la portée du combat qu'il définit en conclusion comme l'affrontement idéologique entre un nouveau système contre l'Ancien Régime européen. Il avait précédémment observé que la manifestation de 1989, si peu convaincante qu'elle ait été, avait renoué avec l'idée française de nation fondée sur la citoyenneté - ouverte - et l'universalité.

Telle est la conclusion de cette claire synthèse étayée sur une vaste bibliographie. Annie CRÉPIN

Quelques pages d'un manuscrit sous la Terreur en Béarn, 1793-1794, documents inédits des Ärchives des Basses-Pyrénées publiés par Joseph Lochard, Nîmes, C. Lacour, 2001,220 p.

Il convient de saluer les efforts de l'éditeur nîmois C. Lacour qui a entrepris de redonner aux lecteurs certains ouvrages sur la période révolutionnaire dans la France méridionale, dont ce livre publié par Joseph Lochard en 1893.

Ce dernier n'a pas toujours eu la main heureuse dans les associations des textes présents dans son recueil. Ainsi, aux pages 7-15, sous le titre «Le Comité de salut public», il mentionne en vrac la création du Tribunal révolutionnaire, la circulaire adressée aux comités de surveillance après le vote du décret du 14 frimaire an II, puis il commente : «Ce programme établi, la Convention nationale envoya des représentants du peuple dans les départements pour tenir la main aux mesures de surveillance et de salut public. En mars 1793, les conventionnels qui furent envoyés en mission extraordinaire auprès de l'armée occidentale [sic] étaient: Pinet, Cavaignac, Garreau [sic], Baudot, Dartigoeyte, Chaudron, Rousseau [sic], Carnot, Féraud, Isoard, Lamarque et Monestier (du Puy-de-Dôme)». Outre les erreurs dans les noms des conventionnels (Chaudron-Roussau qui se dédouble en un Chaudron et un Rousseau !) et les aberrations dans la liste des représentants en mission, la chronologie est superbement ignorée, l'auteur mélangeant le printemps et l'hiver 1793!

Toutefois, en dépit de ces nombreuses erreurs, dont je ne cite ici qu'un exemple, Joseph Lochard a rassemblé dans cet ouvrage des documents très intéressants, notamment sur les différentes autorités constituées et les autorités révolutionnaires locales, leurs relations avec les représentants du peuple en mission et la politique mise en œuvre 
par ceux-ci. La seconde partie du livre est à cet égard la plus digne d'intérêt, car l'auteur nous livre un compte rendu rédigé par Dulaut fils, agent national du district de Pau, à propos des années 1793-1794 (ce compte rendu est approuvé par les autorités du district le 5 brumaire an III). Ce texte est un passionnant document sur la France en Révolution, tant pour la description de l'effort de guerre supporté par les citoyens que pour la rhétorique utilisée. Citons deux exemples de cette dernière :

"Tout a été utilisé par la révolution, les débris de l'ancien gouvernement des rois et des prêtres ont été purifiés et ont servi à faire aller la nouvelle machine politique. Les paperasses de la féodalité orgueilleuse se sont converties en beau papier, marqué du sceau du républicanisme. Les cloches, que le fanatisme agitait pour frapper l'air de sons tristes et lugubres, se sont fondues en canons; les livres d'église, portant les rêveries des prophètes, ont fourni des gargousses, et dans les décombres des temples de la divinité, des charlatans de Rome, les Français devenus libres, philosophes, ont trouvé la foudre, avec laquelle ils écrasent leurs ennemis. "

"Les voitures de luxe étaient des lits mouvants, que l'orgueil, la molesse et l'oisiveté avaient inventés pour les grands de la terre. Ceux-ci ont disparu, leurs voitures ont resté. Aujourd'huy de pauvres sans-culottes blessés ou malades en profitent. C'est en carrosse qu'ils vont à l'hôpital, et c'est l'humanité et la reconnaissance nationale qui tirent un parti si honorable de ces dépouilles brillantes de la grandeur qui s'est éclipsée. »

Le compte rendu de Dulaut, tout entier empli de ce type de démonstrations, est fort heureusement livré au lecteur sans les commentaires de Joseph Lochard. Il s'agit ainsi d'un matériau brut dans lequel chacun pourra à sa guise puiser des extraits pour illustrer tel ou tel aspect de la vie locale en l'an II.

\title{
Michel BIARD
}

\begin{abstract}
Abbé CAU-DURBAN, La période révolutionnaire à Castelnau-Durban (Ariège)(17901802), Nîmes, C. Lacour, 2001, 56 p.

Cette petite plaquette procède du même principe que l'ouvrage évoqué dans le compte rendu ci-dessus : rééditer des ouvrages devenus presque introuvables. Toutefois, la comparaison s'arrête là, car il ne s'agit pas avec le livre de l'abbé Cau-Durban de la publication d'un document original, mais d'une chronique de la Révolution vécue à l'échelle locale, dans une commune de l'Ariège qui comptait alors un millier d'habitants.
\end{abstract}

L'intérêt de l'ouvrage réside donc avant tout dans la prose violemment hostile à la Révolution de l'abbé Cau-Durban, qui tient à s'inscrire dès ses premières lignes dans le sillage de Joseph de Maistre. Si l'on excepte quelques points spécifiques (notamment la question des biens communaux, évoquée aux pages 18-20), ce petit livre retiendra surtout l'attention par ses "morceaux choisis». Je n'en citerai qu'un seul extrait : "Nous voici en 1793, en pleine dictature de la Montagne. Sur la place de la Révolution, à Paris, on venait de jeter la tête du roi martyr à la démagogie, comme on jette une pâture sanglante aux fauves des arènes. Maintenant, plus de frein! Le despotisme et l'anarchie en délire vont faire leur ouvre de brigandage : le culte aboli, les églises pillées, fermées ou profanées par des saturnales, la loi des suspects alimentant les prisons et les guillotines, les biens communaux livrés aux affamés [...] partout le désordre, le pillage, la mort. Voilà le beau régime que la Terreur va imposer à la France!»

Brisons là. On l'aura compris, la découverte de ce petit livre est davantage conseillée à ceux qui s'intéressent à l'historiographie de la Révolution française qu'à ceux qui se passionnent pour son histoire. Aussi laisserai-je la conclusion à l'abbé Cau- 
Durban, car il résume bien le fond de sa pensée politique en une petite phrase, à propos du maire installé en prairial an VIII : «... le Pouvoir, quelle que fut son étiquette, pourvu qu'il eût un programme de répression contre l'anarchie, était assuré de son concours. "

Michel BIARD

Charlotte GoËTZ, Marat en famille, la saga des Mara[t], Bruxelles, Pôle Nord, coll. « Chantiers », 2001, 2 vol., 271+322 p.

La dernière livraison de la collection «Chantiers» s'inscrit dans la lignée des précédentes. Elle permet de suivre les pérégrinations des ascendants et collatéraux de Marat avec une précision tout à fait remarquable. Jean, le père, Pierre et David les frères, Louise la mère ont fait entre autres l'objet d'enquêtes minutieuses et toutes les pièces retrouvées pour étayer les résultats de cette enquête sont mises à la disposition des lecteurs. Certains de ces résultats sont tout à fait intéressants y compris pour ceux que ne passionneraient pas exclusivement pour la destinée individuelle de Jean-Paul Marat : ainsi le travail de Jean Mara pour la STN, illustration assez rare du fonctionnement de cette entreprise capitale du temps des Lumières, mélange de relations somme toute assez personnelles entre Jean Mara et Ostervald; les procédures permettant l'implantation de la STN sont ici illustrées par un exemple précis. De même, le parcours de David vers la Russie et l'ouverture des archives de Saint-Petersbourg permettent de mieux mettre en évidence, à travers un cas concret, les filières de la promotion sociale par la culture au temps des Lumières - ainsi que les difficultés qu'elle présente.

Il s'agit évidemment ici d'une enquête d'une érudition très pointue. Il s'agit également d'un travail reposant sur le postulat de l'absolue vérité de tout ce qu'a pu écrire Marat sur lui-même; reposant également sur celui, trivial, selon lequel «les chiens ne font pas des chats": et voici tous les Mara incompris, courageux, fermes, francs, sincères. Dans la mesure où l'on accepte ces deux postulats - ou dans la mesure où à tout le moins, on ne les considère pas comme invalidant l'entreprise tout entière -, ce double volume est d'une importance capitale pour l'historiographie maratiste.

Olivier COQUARD

Philippe RIVIALE, L'impatience du bonheur. Apologie de Gracchus Babeuf, Paris, Payot et Rivages, Critique de la Politique Payot, 2001, 275 p.

Philippe Riviale, bien connu pour ses travaux sur des penseurs du XIXe siècle, notamment Tocqueville et Charles Fourier et qui a déjà publié un essai sur la Conjuration (1994), propose dans ce livre une relecture des textes de Babeuf. Trois parties tentent avec bonheur de rendre la parole effacée du Tribun, d'abord grâce à son journal, puis en suivant les développements de la Conjuration des Égaux, à travers la défense de Babeuf enfin, et celle des principaux accusés lors du procès devant la Haute Cour de Vendôme. Ce faisant, il rompt avec l'historiographie marxiste qui faisait de Babeuf un précurseur de l'idée communiste, même s'il utilise les textes publiés avant lui par des historiens du babouvisme comme Victor Daline. La démarche est intéressante, car si la Conjuration des Égaux a fait couler beaucoup d'encre, la figure de Babeuf demeure énigmatique; l'analyse des textes de la période trouble de Thermidor et du Directoire a le mérite de lui redonner sa singularité.

La parole du Tribun du peuple émerge quand tous les autres grands tribuns se sont tus, pour refuser les effets de la dé-révolution et l'abandon des principes républicains et démocratiques. Babeuf s'oppose au nouveau cours politique de manière intransigeante, sans crainte de se mettre en avant, construisant sa propre image par son discours radical, celle du proscrit et du futur martyr de la liberté. Le courage de l'homme libre se mesure à la puissance de ses adversaires (p. 89). Babeuf n'est pas un politique et n'accepte pas de se rallier à l'opinion des républicains du jour; il refuse le compromis que 
tentent d'autres démocrates du Directoire, une position qui fait à son sens le jeu du gouvernement en isolant le peuple (p. 60). Il croit encore à l'urgence d'une nouvelle révolution et s'en prend au dictionnaire des puissants, à l'inversion du sens des mots (anarchistes, factieux). Mais force est de dire que si l'exemple du vertueux Goujon peut conforter le courage des âmes fortes opposées à la tyrannie, après le désastre de Prairial, Babeuf se fait des illusions sur la possibilité d'une véritable régénération et sur la capacité de résistance populaire $\left(n^{\circ} 36\right.$ du Tribun du peuple) : égalité, vertu, liberté, la république démocratique est plus que jamais avenir.

En suivant très précisément dans la deuxième partie, à travers la correspondance et les pièces saisies par la police, les discussions entre les principaux membres de la Conjuration, Darthé, Buonarroti, Germain et d'autres, Philippe Riviale montre, toujours en privilégiant les textes, à quel point Babeuf est seul. Si les républicains démocrates s'entendent sur les principes et les buts de la Révolution, ils sont loin de s'entendre sur les moyens de parvenir à un meilleur ordre social. La pensée de Babeuf est révolutionnaire à la manière de celle de Rousseau, ce que montrent ses échanges avec Bodson sur les robespierristes ou avec Antonelle à propos de la propriété, "par le moyen de laquelle chaque famille est une république à part» (p. 117). Comme Rousseau avant lui, Babeuf refuse cette figure de la république : il n'y a de république que celle des citoyens égaux, de liberté et de bonheur que la liberté et le bonheur pour tous. La première instruction du directoire secret, remarquablement commentée, est un autre texte capital sur la pensée de Babeuf. Il se clôt sur les principes du Contrat social pour résoudre dans la pratique ce «beau problème» de la liberté républicaine : "que chacun de nous ne dépende que des institutions et des lois; et qu'aucun de nous ne tienne personne sous sa dépendance » (p. 148).

La dernière partie commente les arguments des accusés devant la Haute Cour de Vendôme, pour redonner à chacun sa place et sa vérité dans la Conjuration, en suivant les comptes rendus et débats imprimés du procès. On suit bien l'auteur dans sa démonstration quand aux intentions du Directoire de donner, contre Babeuf, de la publicité à l'affaire pour attiser les peurs. Reste que les débats, les interrogatoires et les défenses faillirent bien faire tourner le procès public à l'avantage des accusés. Ceux-ci réaffirment inlassablement leurs principes, plaidant sur l'intention vertueuse d'insurger les esprits en vue d'un meilleur système politique. Que Babeuf s'ingénie à tourner en dérision les conclusions des « experts » en écriture, ou que Buonarroti et Germain récusent le témoin Grisel sur l'incohérence de ses déclarations, il s'agit de ruiner les preuves de la réalité d'une conspiration, de dissiper le fantôme effrayant de l'insurrection. L'apologie de Babeuf se révèle être parfois injuste pour ses compagnons de route, pour des républicains comme Darthé ou Buonarroti. Ce dernier appuie sa défense sur la théorie des peuples libres : "Ce qui distingue essentiellement un peuple libre d'un peuple esclave, c'est que chez le premier l'émission de sa volonté est l'ordre tranquille et habituel, tandis que chez le second, c'est un effort pénible qui passe souvent pour révolte. C'est sous ce dernier point que je considérais l'état du peuple français [...]» (p. 204). Les babouvistes développent les thèmes républicains quand le républicanisme n'a plus cours, et que les mots ont changé de sens, ainsi de la dictature préconisée par Darthé, non celle dont parlent les thermidoriens mais la dictature au sens néo-romain. Le discours babouviste reprend contre le discours du pouvoir et l'empire usurpé des mots le vocabulaire de l'humanisme civique, sans pouvoir briser le cours du discours dominant où le changement conceptuel est un des ressorts cachés de l'argumentation. L'étude du vocabulaire républicain de Babeuf serait éclairante.

Babeuf était une "plume", il est condamné à mort pour ses écrits, ainsi que Darthé en vertu de la loi du 27 germinal an IV, pour avoir provoqué au rétablissement de la Constitution de 93. « Je ne parlerai plus, dit Babeuf après le verdict. La mort pour des écrits! " En conclusion de ce livre d'une grande richesse, les deux dernières lettres de Babeuf, à Félix Lepeletier et aux siens, assez poignantes en elles-mêmes pour découra- 
ger tout commentaire : «Mes amis, j'espère que vous vous souviendrez de moi, et que vous en parlerez souvent. J'espère que vous croirez que je vous ai tous beaucoup aimés. Je ne concevais pas d'autre manière de vous rendre heureux que par le bonheur commun. J'ai échoué ; je me suis sacrifié ; c'est aussi pour vous que je meurs ». Réduire les inégalités que génère la société marchande, le problème est toujours d'actualité, même s'il se pose de nos jours à une toute autre échelle qu'au temps de Babeuf. Merci à l'auteur d'avoir si bien su évoquer une figure héroïque de la Révolution qui pensait pouvoir réveiller l'espérance du bonheur et de ces choses très anciennes nommées égalité, justice, liberté.

Raymonde MONNIER

Stefano Solimano, Verso il Code Napolèon. Il progetto di Codice civile di Guy JeanBaptiste Target (1798-1799), Milan, Giuffré, 1998, 427 p.

Tel l'archéologue mettant au jour une pièce rare ou un trésor enfoui, l'historien peut, mais beaucoup plus rarement, trouver dans la poussière des archives un document d'autant plus précieux qu'il était ignoré. C'est ce qui est arrivé à Stefano Solimano, jeune juriste milanais familier de nos Archives nationales, dont la ténacité à dépouiller les papiers du ministère de la Justice a été féconde. N'y a-t-il pas découvert sous la cote BB 299 réunissant des pièces disparates, un projet de codification du droit civil dont personne ne connaissait l'existence, mais qui peut être attribué sans l'ombre d'un doute au « citoyen Target »? Quant à la date de rédaction du manuscrit (un cahier de 22 pages grand format), l'étude de son contenu comparé aux règles du droit positif contemporain, conduit $S$. Solimano à la situer à la fin du Directoire, donc assez longtemps après le troisième projet officiel présenté devant le Conseil des Cinq-Cents par Cambacérès (prairial an IV-juin 1796). Le texte découvert a très vraisemblablement été composé en l'an VII, en tout cas avant le coup d'État et, par conséquent, avant que Jacqueminot, au tout début du Consulat, présente son propre projet partiel de Code civil. D'ailleurs, outre le projet de Target, sont publiés en appendice, des fragments jusqu'alors inédits des travaux de Jacqueminot. Mais l'intérêt de l'ouvrage ne réside pas seulement dans cette publication de textes (qui remplit seulement quelques dizaines de pages sur un total de plus de 400 ). L'auteur, avec talent et originalité, l'insère dans le vaste contexte de «la normalizzazione della vita publica e civile» et du désir d'en finir avec la Révolution.

La première partie (pp. 17-157) - qui concerne d'ailleurs aussi bien le droit pénal que le droit civil - expose les fondements idéologiques et les " racines thermidoriennes " de la codification, de la chute de Robespierre au début de l'Empire. Là, dans une certaine mesure, $\mathrm{S}$. Solimano renouvelle la question, faisant une large part, non seulement à l'apport des Idéologues mais aussi des penseurs utilitaristes, les idées de Bentham ayant été l'objet d'une « réception précoce » en France, à la suite de la traduction et de la publication à Genève, dès 1796, dans la Bibliothèque britannique, de certaines de ses ouvres, notamment de l'Introduction aux principes de morale et de jurisprudence. D'une manière générale, sont finement analysées les influences culturelles, y compris celles des avatars du jansénisme, exercées sur les juristes de la Convention thermidorienne et des Conseils du Directoire, ainsi que sur « Napoléon et son équipe ".

Le décor idéologique et politique étant ainsi brossé, la deuxième partie (pp. 158-232) est consacrée au " parcours spirituel » de Target, « de liillusion au désenchantement ». Ici aussi l'auteur apporte des éléments nouveaux puisés dans les archives, qui éclairent la personnalité du personnage et ses réactions. Sont analysés tour à tour les moments marquants de la vie publique du jurisconsulte : années de formation de l'avocat, puis du magistrat nancéien, ébloui par les Lumières; débuts de la Révolution lorsque Target, membre du Comité de constitution, siège à la Constituante et est élu juge du cinquième arrondissement, à Sainte-Geneviève ; épisode obscur du procès du roi et de la maladie diplomatique de l'avocat pressenti; acceptation prudente du titre de 
secrétaire du Comité révolutionnaire de la section de l'homme armé (novembre 1793); le Consulat, enfin, alors que le juriste chevronné est nommé au Tribunal de cassation où il préside la section civile (an VIII), qu'il participe à la fondation de l'Académie de législation, est nommé au Tribunat (1802) et à l'Institut (1803). Bref, au cours des dernières années de sa vie (il mourra en 1806), Target est devenu un «parfait technocrate napoléonien ».

La troisième partie (pp. 233-346) est composée en contrepoint de la première. Elle est consacrée au projet de Code civil lui-même et permet de voir comment les présupposés idéologiques de l'auteur se traduisent en termes juridiques et institutionnels. Si le texte est court, divisé en 272 articles dont certains fort laconiques, et rappelle ainsi les deux premiers projets du Comité de la Convention, son inspiration le rapproche non seulement du troisième (1796) mais, surtout, de celui qui aboutira en 1804. Il apparaît un peu comme un canevas, purement officieux, de la codification à venir, pour le moins comme un jalon, le dernier, dans la longue marche, faite de renoncements aux idéaux révolutionnaires de l'an II, qui s'achèvera au début de l'Empire. Si l'adoption et le divorce, y compris par consentement mutuel et incompatibilité d'humeur, sont maintenus, l'autorité est renforcée dans la famille au profit de celui qui est «chef de la maison conjugale et du ménage », la femme mariée étant de nouveau considérée comme incapable. Quant au droit des biens, des obligations ou des successions, le projet exprime les grandes lignes de l'individualisme libéral. Target n'avait-il pas, en 1789, défini la propriété comme «le droit qui appartient à chaque homme, d'user et disposer exclusivement de certaines choses ", termes qui annoncent la célèbre formulation de l'article 544 du Code civil?

On aura compris que l'ouvrage de Stefano Solimano déborde largement les contours d'une monographie biographique, ou de la sèche analyse d'un projet inédit. Ce livre élégant, publié dans la collection de l'Institut d'histoire du droit italien de Milan, touche aux grands problèmes actuels de l'historiographie révolutionnaire.

\section{Jean BART}

Jacques ADÉlAÏDE-MERLANDE, R. BÉlÉNUS, et F. RÉGENT, La Rébellion de la Guadeloupe, 1801-1802, Gourbeyre, Guadeloupe, 2002, 355 p.

Le conseil général, la Société d'histoire de la Guadeloupe et les Archives départementales se sont associés pour concevoir et éditer ce livre qui commémore la révolte de 1802. Les auteurs redonnent la parole aux acteurs et font revivre Delgrès, Pélage, Ignace et leurs compagnons en se servant de documents en majeure partie extraits des archives locales. Les textes officiels se mêlent aux lettres privées, les dépêches ministérielles aux rapports militaires, les proclamations aux extraits de journaux.

Les imprimés ou les manuscrits fidèlement reproduits sont accompagnés d'analyses succinctes, de références archivistiques, de notes infrapaginales et de commentaires. Une chronologie où s'inscrivent les événements qui eurent lieu en Guadeloupe, à Saint-Domingue ou en France, des cartes fort bien faites, un petit dictionnaire biographique et une bibliographie sommaire facilitent encore la lecture.

Les textes sont répartis en trois parties:

1. Le renvoi de Lacrosse et le gouvernement de Pélage, 21 octobre 1801-5 mai 1802.

2. L'expédition Richepance et ses conséquences.

3. Le retour à l'ordre ancien. Le livre est rédigé par des universitaires guadeloupéens : MM. Adélaïde-Merlande, Bélénus et Régent, auteur d'une thèse remarquable sur les esclaves, les hommes libres et citoyens de couleur en Guadeloupe de 1789 à 1802, bientôt publiée. Au-delà des enseignants et des étudiants, le livre s'adresse à un large public. Un beau travail collectif au service de la mémoire d'hommes qui voulurent : «Vivre libre ou mourir ». 
Intendants et préfets dans le Nord-Pas-de-Calais (XviIe-XXe siècle), actes du colloque d'Arras (2000), textes réunis par Alain LOTTIN, Annie CRÉPIN et Jean-Marc GuISLIN, Arras, Artois Presses Université, 2002, 344 p.

Le bicentenaire de la création de l'institution préfectorale a fort logiquement donné lieu à de nombreux colloques et journées d'étude. Parmi ceux-ci, le colloque d'Arras occupe une place beaucoup plus importante que ne pourraient le laisser à penser les bornes géographiques choisies. D'une part l'institution a été envisagée dans ses racines comme dans son devenir pour mieux "... appréhender le phénomène administratif dans le long terme ", d'autre part certaines des communications sont d'une telle richesse que l'exemple septentrional peut donner à penser pour l'ensemble du territoire national.

L'ouvrage comporte quatre parties : la première est réservée aux intendants; la seconde étudie la création des préfets et les premières années de l'institution (1800-1814); la suivante concerne l'action préfectorale de 1814 à 1871 ; enfin la dernière comprend des communications sur la Troisième République. Ce sont bien sûr avant tout les deux premières qui retiendront l'attention des lecteurs des A.H.R.F.

Le premier intendant évoqué ici l'est sous la plume d'Alain Lottin. Celui-ci nous livre un portrait, assez classique, de Michel Le Peletier de Souzy, intendant choisi pour la toute nouvelle intendance de Flandre en 1668 (il demeure à ce poste jusqu'en 1683). L'auteur rappelle les attributions de ce personnage qu'il définit comme "l'homme-clef du pouvoir central ", mais il met surtout l'accent sur le fait que l'intendant de Flandre doit avant tout s'efforcer d'attacher de manière définitive la nouvelle province au royaume. Classique également l'étude que consacre Charles Engrand aux intendants de l'Artois sous le règne de Louis XIV. On y découvrira l'itinéraire des intendants successifs, l'analyse de leur correspondance, mais aussi leur volonté de dialoguer avec les "groupes sociaux » présents dans les institutions provinciales et locales.

Les deux études les plus intéressantes sont toutefois, à mon sens, celles de Philippe Guignet et de René Grevet, dans la mesure où leurs conclusions ont une portée beaucoup plus large. Le premier de ces auteurs évoque le portrait de Sénac de Meilhan pour mieux aborder les fonctions d'intendant au temps de Louis XVI. Cet «intellectuel en politique " a rédigé de nombreux ouvrages, dont l'un est essentiel dans l'élaboration du portrait collectif des intendants : Du Gouvernement, des mours et des conditions en France avant la Révolution, publié dans l'émigration, à Hambourg, en 1795. Cet écrit est à la base de nombreuses idées reçues sur les intendants : les «intendants tracassiers, imbus de leur autorité du temps de Colbert [...]» y sont opposés à leurs successeurs "éclairés » du règne de Louis XVI. Sénac de Meilhan, qui affiche des positions libérales en certains domaines (par exemple à propos de la libre circulation des grains), n'est cependant «... nullement un adepte du moins d'État sur le plan politique " et considère que l'intendant doit être «... fondamentalement l'homme du roi et l'homme de l'État impartial, attentif aux besoins du peuple [...] ». C'est d'ailleurs au nom de cette conception qu'il s'oppose à l'affaiblissement du pouvoir royal et notamment au «... transfert de prérogatives opéré au nom du libéralisme aristocratique ».

L'homme est également présent dans la communication de René Grevet, qui porte sur « la fin des intendances et la transition administrative dans les provinces septentrionales (1789-1790) ". L'institution est décrite comme déclinante, affaiblie dans son rôle traditionnel et concurrencée par des pouvoirs rivaux, notamment par les États provinciaux (sur ceux-ci, on se reportera au récent et convaincant travail de Marie-Laure Legay). L'attitude des trois intendants d'Amiens, Lille et Valenciennes face aux ruptures de l'été 1789 est assez semblable : à Lille, Esmangart démissionne le 8 septembre sous prétexte d'ennuis de santé; à Amiens, d'Agay de Mutigney quitte son poste pour Besançon, le berceau familial, à la fin de juillet ou au début d'août, puis revient à l'automne et exerce ses fonctions jusqu'en août 1790; enfin, à Valenciennes, Sénac de Meilhan adopte une attitude de réserve dès l'automne 1789, avant d'émigrer dans l'été 
1790. Comme ailleurs, ce sont donc les subdélégués généraux, nommés afin de réaliser la passation des pouvoirs avec les nouvelles administrations, qui assurent tant bien que mal la continuité de l'État pour l'administration, la fiscalité et la sécurité publique.

Mais au-delà de ces détails livrés sur trois intendances de la France septentrionale, l'étude de René Grevet est passionnante par sa réflexion sur l'image des intendants en 1789. S'il rappelle à son tour comment le rôle des intendants a pu être réévalué par les historiens (notamment dans la lignée des travaux de François-Xavier Emmanuelli), il souligne surtout que le problème central est ailleurs : «N'est-ce pas réduire l'analyse en distinguant d'un côté, et il y en eut, les bons intendants, bienfaisants et entreprenants, et de l'autre, on en connaît aussi, les mauvais, négligents, dissipateurs et despotiques? Remarquons que les uns comme les autres furent unanimement condamnés dans les cahiers de doléances et impitoyablement rejetés. Impossible donc de s'en tenir exclusivement à une réhabilitation post mortem en tenant compte de l'œuvre importante accomplie par les intendants car l'on s'enfermerait immédiatement dans une démonstration contredite par le rejet catégorique de 1789. Impossible également de s'en tenir uniquement à la thèse des abus de l'institution pour expliquer le rejet et la disparition des intendances, car ce serait promouvoir un principe causal, trop général, qui ne résisterait guère à de nombreuses exceptions. Il semble beaucoup plus judicieux de déplacer le périscope historiographique vers l'inadaptation croissante de l'organisation administrative datant de l'ère louisquatorzienne».

Ce qui anéantit les intendants, avec le vote de la Constituante du 10 décembre 1789 , c'est fondamentalement cette incapacité de l'État à s'adapter et à offrir aux élites provinciales une plus large représentation afin de «... favoriser leur intégration dans les rouages administratifs de l'État ». Blocages institutionnels, blocages politiques, blocages économiques et sociaux se conjuguent ainsi pour perdre le «régime». Quelque part, le débat sur le rôle «centralisateur » des intendants s'en retrouve relégué à l'arrière-plan, d'autant que Marie-Laure Legay a par ailleurs montré que la lutte entre eux et les États provinciaux était (au moins dans l'exemple septentrional) davantage un conflit pour exercer le rôle d'agents du roi que le résultat de velléités « décentralisatrices ».

Avec une telle communication pour clore la partie réservée aux intendants, on aurait pu souhaiter qu'une place soit faite aux expériences révolutionnaires, aux procureurs généraux syndics, aux représentants du peuple en mission, aux commissaires centraux du Directoire. Les représentants élus dans les Assemblées successives de la Révolution n'ont en effet jamais cessé d'avoir à l'esprit le contre-modèle des intendants, et il aurait sans doute été très intéressant de se pencher sur la décennie 1789-1799 pour mieux comprendre l'apparition de l'institution préfectorale. Pourtant, les thèses de Tocqueville imprègnent encore en partie l'historiographie et le colloque passe sans transition de la fin des intendances (1789-1790) à la création des préfets (1800).

Ceux-ci font l'objet d'une demi-douzaine de communications qui portent toutes sur la période du Consulat, de l'Empire et de la Restauration. Jean-Paul Bertaud ouvre cette partie par une réflexion sur le rôle du préfet comme «maître de cérémonies ». Il décrit, avec beaucoup d'humour, les mille et un conflits de préséances rencontrés par les préfets, avec les notables qui rechignent, avec les juges qui arguent de leur indépendance vis-à-vis du pouvoir politique, et plus encore avec les militaires qui n'entendent point céder les places d'honneur aux " pékins ", fussent-ils préfets. Le décret impérial du 24 messidor an XII (13 juillet 1804) met fin aux débats, à défaut de mettre fin aux querelles, en établissant préséances et honneurs civils et militaires dans les cérémonies publiques. Avec ce texte, les préfets n'apparaissent désormais plus qu'en treizième position et les sous-préfets à la dix-neuvième place! Ce que voit avant tout le peuple, à présent réduit au rang de spectateur, «... c'est une autorité militaire qui, dans son désir légitime de conserver la place que ses sacrifices lui ont value, présente ses valeurs comme le bien suprême [...] ». À ce jeu là, le préfet ne risquait guère de l'emporter. 
Trois autres communications livrent avant tout des portraits d'administrateurs : celle de Vincent Cuvilliers sur les cinq premiers préfets du Pas-de-Calais, entre 1800 et 1815 (avec notamment le baron de La Chaise, préfet en 1803, qui se rallie sans états d'âme aux Bourbon en 1814, avant d'esquisser un nouveau et vain retournement au moment des Cent-Jours; mais aussi avec l'ancien Montagnard André Dumont, qui fut représentant en mission et un éphémère préfet en mai-juin 1815); celle de Karine Van Wynendaele sur leurs quatre homologues du Nord entre 1815 et 1830 ; enfin celle de Matthieu de Oliveira sur Bottin, secrétaire général de la préfecture du Nord de 1802 à 1814 , l'un de ces hommes essentiels dans les rouages des préfectures et ici le personnage clef dans l'établissement des Annuaires statistiques du département.

Philippe Raxhon évoque, quant à lui, les préfets des «départements réunis », à travers le cas liégeois. Ici se succèdent sous le Consulat et l'Empire deux hommes : Desmousseaux, qui obtient au printemps 1800 le département de l'Ourthe, alors qu'il aspirait à celui... des Bouches-du-Rhône ; puis son successeur, Micoud d'Umons, d'avril 1806 à janvier 1814. L'auteur montre combien leur activité a été fondamentale pour jeter les bases des «services publics ", favoriser la diffusion du savoir et des inventions, mettre en place «... un creuset définitif pour l'apprentissage de la gestion des affaires publiques dans un XIX siècle laïcisant, et dans le cadre d'une Belgique indépendante et unitaire $»$.

Restent enfin deux interventions qui mettent en valeur certaines tâches précises des préfets : celle de Pierre-François Pinaud à propos du personnage à part qu'est alors dans un département le receveur général, sorte de "préfet financier» avec lequel le préfet (en théorie son supérieur) a établi une sorte de modus vivendi et ne joue guère sur les rapports d'autorité; et surtout celle d'Annie Crépin qui rappelle le rôle majeur du préfet dans cette "machine conscriptionnelle " qu'il cherche sans cesse à améliorer. On s'attendrait a priori au portrait d'un homme qui se soucie surtout de faire exécuter la loi et de réprimer tout ce qui pourrait l'entraver. Or le mérite du texte d'Annie Crépin est de nous décrire un préfet qui, loin d'être un banal exécutant, sait "moduler", «tempérer», dénouer les situations les plus difficiles, en bref joue un rôle tout en nuances, mais efficace, pour enraciner la conscription.

Si l'on ajoute qu'un tiers environ des communications proposées à ce colloque n'entrent point dans mon compte rendu, car elles concernent le Second Empire et la Troisième République, il me semble que tout discours supplémentaire serait vain pour vanter les mérites de cet important ouvrage. Il faut donc impérativement le lire !

\title{
Michel BIARD
}

\begin{abstract}
Napoléon, Stendhal et les romantiques, l'armée, la guerre, la gloire, Actes du colloque organisé par le Musée de l'Armée, textes réunis par M. ARrous, Paris, Eurédit, 459 p.

Le colloque organisé par le Musée de l'Armée rappelle dans sa démarche générale celui de Clermont-Ferrand de 1985 sur La bataille, l'armée et la gloire. Les communications présentées s'intéressent plus particulièrement au travail de création ou d'interprétation de la légende napoléonienne par Stendhal et par la génération des romantiques. En un temps où la bourgeoisie triomphante adopte le mot d'ordre « Enrichissez-vous", les romantiques fascinés par l'armée de Napoléon et par la gloire des batailles en entreprennent une véritable mythification. Certains, rejetant le culte de Napoléon « multiplicateur de l'enthousiasme ", conservent une attitude critique.
\end{abstract}

Une première partie regroupe des communications portant sur le thème: "Figures de l'officier et du soldat, vie en campagne, vie en garnison». T. Oswald présente un Balzac qui, tout au long de son œuvre, étudie comme un anthropologue les héros de la légende. V. Laisney décrit un Charles Nodier empli tout à la fois de haine et d'amour à l'égard de Napoléon qui frustre le poète de sa gloire. P.-M. Nadeau, faisant revivre la figure du demi-solde chez Coignet et Stendhal, s'interroge sur la gloire, « deuil 
éclatant du bonheur ». Pour J.-T. Nordmann, Taine, transformant Napoléon en héros romantique, se projette dans sa représentation de l'Empereur bien plus qu'il ne se soumet à elle. Quant à P.-L Courier, il est, sous la plume de J.-P. Lautman, le grand juge d'une armée qui «sans humeur et sans honneur " récolte des lauriers peu estimables. C. Réquéna, étudiant les personnages militaires dans l'œuvre de Mérimée y trouve associés Mars et Vénus. J.-C. Yon montre comment Offenbach accentuant jusqu'à la caricature les stéréotypes militaires, raille les valeurs de l'armée. N. Boussard dans "Odieux de la paix, apologie de la guerre et nostalgie de la gloire» découvre un Stendhal pour qui la passion de la gloire est une vertu anachronique.

La partie consacrée à " La gloire, l'exploit, le courage » rassemble des communications sur la vision qu'en eurent les officiers et les soldats de l'armée de Napoléon (G. Bodinier), sur la description par Balzac de la «plus horrible des batailles»: la Bérézina (M. Di Malo), sur l'ambition ou la gloire en deuil (M. Guérin), sur l'épisode du Saint-Bernard dans la Vie de Henry Brulard (X. Bourdenet), enfin sur l'historiographie militaire de la bataille de Waterloo et son influence sur Stendhal et Hugo (J. Garnier).

Le thème «Batailles perdues » est illustré par N. Piétri, «Sedan, la défaite de 1870 et son retentissement », par V. Rambaud, «Barrès et le Professeur d'énergie », par C. Herzfeld, "Chronique d'une défaite annoncée" (celle de 1870 par O. Mirbeau), par E. Roy-Reverzy «La Débâcle de Zola, l'écriture de la guerre ou la rhétorique de l'Histoire ". Enfin, M. Crouzet, dans " Napoléon et Stendhal, gloire militaire et gloire littéraire ", montre comment pour Stendhal "l'égalité, c'est l'égalité dans l'honneur; le peuple s'est accru en nombre, en aisance, en moralité, il a surtout accédé à la gloire et à l'honneur".

Un livre qui saisit le regard croisé des historiens et des littéraires sur l'ambition et l'héroïsme, sur la gloire et le deuil, "sous le signe nostalgique ou prophétique de Napoléon ".

\section{Jean-Paul BERTAUD}

Terminée la Révolution..., (Actes du IVe colloque européen de Calais, 26-27 janvier 2001), textes réunis par Michel BIARD, Bulletin des Amis du Vieux Calais, 2002, 252 p.

Dans son introduction, Michel Biard donne sens et signification au projet assumé par cet important colloque de Calais : «Terminée la Révolution... Trois mots associés dans un ordre voulu et dans un ordre qui n'est point celui de la tradition. En effet, l'usage a plutôt été, depuis un peu plus de deux siècles, d'évoquer la question de la Révolution à terminer ou bien de gloser sur la Révolution terminée... sans points de suspension ». La lecture de ces actes témoigne de l'importance de l'enjeu et, dans le cadre d'une très libre expression des communicants, de l'actualité renouvelée du thème ainsi posé.

Quatre axes de réflexion ont successivement été suivis, donnant au colloque et à ses Actes une incontestable cohérence et une parfaite lisibilité.

En premier lieu est posé le problème des contradictions fondamentales et persistantes entre la «révolution niée » et la « révolution assumée ». Jean-Pierre Jessenne présente successivement le problème des rapports entre l'apparition du mot « révolution et le processus historique singulier qui transforme la France entre 1789 et 1799 et devient une référence mondiale », celui des rapports entre une historiographie des «grands acteurs ou auteurs » et « la révolution vécue par des Français plus ordinaires », celui enfin des analyses elles-mêmes contradictoires qui fleurissent dès le début de l'événement et qui s'inscrivent fortement dans le premier XIXe siècle. C'est au travers de la satire littéraire que Philippe Bourdin propose de tracer le contour de «l'impossible bilan" de ce XVIIIe siècle dont la clôture révolutionnaire peut aussi se lire dans les douze ouvrages, divers et souvent opposés - même s'ils "se rejoignent aussi sur la faiblesse du pouvoir politique»- choisis comme supports de cette communication. Avec 
l'intervention de Jacques-Olivier Boudon sont mis en place les éléments constitutifs des luttes entre "Contre-Révolution « et «recharge révolutionnaire" qui illustrent la période finale de l'Empire, période de conflits violents qui précèdent et expliquent les contradictions qui naissent de la Charte de 1815 et se prolongent pendant tout le cours de la Seconde Restauration. Jean-Claude Caron met en lumière, à propos des Trois Glorieuses, les trois principales "lectures » auxquelles a donné lieu l'événement de juillet 1830 : "révolution-coda" de celle de 1789 avec le spectre de la violence «terroriste " à agiter et à dénoncer, "révolution-appendice " qui justifie aux yeux de certains l'appel à la mise en place d'une république retrouvée et rénovée, "révolution-palimpseste " autour des tenants d'une révolution sociale, coeur de la fondation d'une république véritable.

Dans un deuxième temps, inauguré par le rapport présenté par Annie Crépin sur le thème "grandeur, pouvoirs, légitimité », la période du Consulat et de l'Empire est interrogée autour de la problématique de l'achèvement ou de la rupture par rapport au moment révolutionnaire précédent. Jean-Paul Bertaud montre d'abord dans quelle mesure la volonté personnelle qui initie « la gloire» et le devoir impliqué dans toute la démarche de «l'honneur » trouvent des réalisations à la fois contrastées et variables, notamment dans les Proclamations aux armées et les interventions publiques du grand homme qui « mêlant l'honneur des militaires à la vertu des citoyens [...] les tourne vers la gloire de la Nation révolutionnaire qu'il incarne ». Bernard Gainot s'interroge sur le caractère fictionnel - ou fictif - que le pouvoir législatif présente entre 1799 et la fin de l'Empire : il témoigne de la prééminence croissante du pouvoir exécutif sur un législatif réduit aux travaux désormais secrets des commissions spécialisées d'un Corps législatif asservi au chef de l'État. Josiane Bourguet-Rouveyre remet en cause l'idée d'un étouffement total de la citoyenneté sous le Consulat et l'Empire, en particulier à l'occasion du fonctionnement des assemblées de canton. Avec Natalie Petiteau, c'est le problème des formes d'opposition à la puissance impériale qui est posé : celle des «serviteurs du régime ", au niveau local comme au plan de l'État; celle que prennent « les rébellions contre l'ordre établi "; celle qui s'exprime dans les "actes séditieux " que révèlent les rapports de la police impériale.

Dans un troisième temps, après le rapport introductif de Michel Biard, ce sont des héritages administratifs, sociaux et culturels légués par la Révolution qu'il s'agit. Michel Pertué met en lumière le rôle joué depuis lors par les échelons intermédiaires de ce «modèle administratif français » autour de la loi du 28 pluviôse an VIII qui institua «le couple très inégal de l'administrateur unique et du conseil multiple ", modèle diversement appliqué dans le reste de l'Europe à l'occasion des conquêtes napoléoniennes et dont les effets sur la géographie actuelle de l'administration, au niveau de chaque pays comme à celui de l'Europe sont toujours vivaces. Hervé Leuwers insiste sur les transformations du métier d'avocat qui, après sa suppression à l'époque révolutionnaire, connaît dans le premier XIXe siècle «un renouveau assumé » et retrouve, en la modifiant, l'importance de la profession restructurée au sein de ses barreaux. S'écartant de la thèse d'une suppression définitive des formes de sociabilité vécues sous l'Ancien Régime, Pierre-Yves Beaurepaire témoigne, à travers le cas de la Société philomatique de Paris, du renouveau connu, dès le Directoire, par des sociétés savantes sans cesse plus "professionnalisées" et que l'État se soucie à la fois de contrôler et de développer. Avec Annie Jourdan est abordée la question de l'héritage artistique de la période révolutionnaire : des fêtes de l'Être suprême aux «sujets dignes de la Grande Nation ", l'image de l'artiste peintre de son temps et de ses gloires perdurera, avec des fortunes diverses tout au long du XIXe siècle.

Dans un quatrième temps, six communications ont pour objet de répondre partiellement à la question : que reste-t-il de la Révolution française? D'abord, dans l'immédiat après, Claude Mazauric pose le problème de l'histoire du premier XIXe siècle à l'épreuve du modèle révolutionnaire; Louis Hincker examine les expériences populaires 
de la prise d'armes révolutionnaire au XIXe siècle, notamment autour de l'image du citoyen-combattant à Paris durant le Seconde République; Philippe Minard interroge la notion de libéralisme en économie telle qu'elle peut se définir après les expériences révolutionnaires. Ensuite, dans un passé plus récent, Jean-Clément Martin analyse les formes et les effets de «l'affaiblissement de la rhétorique contre-révolutionnaire »; Bruno Béthouart pose les termes actuels de la problématique des chrétiens face à - ou avec - la Révolution française; Serge Wolikow recherche les références revendiquées par les "mouvements révolutionnaires " du XXe siècle à la Révolution française et l'héritage contrasté assumé par la gauche à l'époque actuelle.

Les Actes de ce colloque se terminent par un bref résumé de la table ronde qui a réuni, sur le thème de "l'idée de Révolution à l'aube du XXIe siècle », avec Jean-Luc Mayaud des historiens aussi divers que Bruna Consarelli, Bronislaw Baczko, JeanPierre Jessenne, Claude Mazauric et Philippe Minard.

Au total, un ouvrage qui permet au lecteur d'une part d'être utilement mis - ou remis - en contact avec les contradictions qu'impose toute approche des bilans de la Révolution française et d'autre part avec les axes novateurs de la recherche historiographique dans le domaine de cette période capitale pour la réflexion sur le temps présent.

Claude COQUARD

Juan Francisco FUENTES, Lluís ROURA eds., Sociabilidad y liberalismo en la España del siglo XIX. Homenaje a Alberto Gil Novales, Lleida, Editorial Milenio, 2001, $348 \mathrm{p}$.

Les livres hommages à un grand historien souffrent souvent d'un défaut majeur : le manque de cohérence. Le lien entre les articles tient moins alors à une thématique ou à une approche méthodologique commune qu'à la manifestation d'un geste d'admiration et d'amitié des auteurs à l'égard du personnage célébré. Ce livre hommage à Alberto Gil Novales, publié à l'occasion de son départ à la retraite, échappe en grande partie à ce défaut. Les seize articles qui le composent sont tous centrés, à une exception près, sur la première grande phase de l'Espagne libérale : 1808-1868.

Douze articles sont consacrés aux thèmes de prédilection d'Alberto Gil Novales : la sociabilité, l'opinion publique et l'histoire de la presse. Deux textes portent un éclairage sur les pratiques politiques à l'échelle locale sous Isabelle II. Lucienne Domergue, avec une grande finesse, tente de donner un sens politique aux messages visuels laissés par le grand muet désenchanté qu'était Goya au travers de sept de ses gravures. L'ouvrage se termine enfin sur un récit et une mise en perspective de la carrière et de l'œuvre d'Alberto Gil Novales. Loin de l'hagiographie, ces 19 pages rédigées par Lluís Roura représentent un matériau passionnant pour l'étude d'une génération d'intellectuels : celle des enfants de la Guerre civile. Dans l'université en ruine du premier franquisme, Alberto Gil Novales s'inscrit dans le groupe réduit des héritiers de la génération d'intellectuels et d'universitaires, issus de la Institución Libre de Enseñenza, qui firent la Seconde République espagnole. En témoignent le choix de ses principaux thèmes d'études jusqu'au milieu des années 1960 (le «régénérationiste » Joaquín Costa et le poète Antonio Machado), ainsi que la correspondance qu'il a entretenue avec l'exilé qu'était alors Alberto Jiménez Fraud, l'ancien directeur de la célèbre Residencia de .Estudiantes_de_Madrid_celle_qui abrita_Dali, García_Lorca... Par la suite, Gil Novales fait partie de ces intellectuels anti-franquistes, démocrates et républicains, qui furent assez vite déçus par la direction prise par la jeune démocratie espagnole, par l'amnésie collective et la plongée dans la société de consommation qui sont loin de l'idéal de citoyenneté qui était celui du kraucisme de ses maîtres, idéal que l'on pourrait assimiler assez sommairement pour un Français ignorant des réalités espagnoles à un mélange de jansénisme, de jacobinisme et de citoyenneté morale tocquevillienne. 
La production d'Alberto Gil Novales est impressionnante : 256 publications scientifiques de 1953 à 2001, sans compter les critiques d'ouvrages, les articles publiés dans la presse... Néanmoins trois chantiers dominent dans cette œuvre foisonnante. La première est constituée par son grand livre sur les Sociétés patriotiques durant le Trienio liberal (1820-1823). Publié en 1975, cet ouvrage est pionnier à plusieurs égards. Avec lui, Gil Novales est le premier à évoquer les formes et les lieux de sociabilités et leur rôle dans la naissance d'une culture politique. Il est aussi le premier à utiliser la prosopographie en histoire politique. On s'étonne d'ailleurs qu'un si grand livre n'ait pas été traduit en français, tant s'imposent les comparaisons avec les sociétés populaires révolutionnaires ou avec les groupes républicains des années 1815-1848. Le deuxième grand chantier ouvert par Gil Novales est constitué par la publication en 1991 du dictionnaire biographique du Trienio liberal. Fruit d'un travail collectif colossal, cet ouvrage devenu une référence propose une courte biographie de tous les Espagnols ayant eu une activité politique de la Guerre d'Indépendance aux années 1840. Alberto Gil Novales est enfin le fondateur et le directeur très actif de la revue Trienio, Ilustración y Liberalismo qui en est à son 36e numéro depuis sa fondation en 1983.

Rien d'étonnant de constater la part dominante occupée par la sociabilité dans l'ouvrage hommage qui lui est consacré au travers d'articles rédigés par les meilleurs spécialistes sur la question. Jean-Louis Guereña offre une remarquable synthèse sur les nouveaux lieux et formes de sociabilités (athénées, cercles, casinos, tertulías et sociétés diverses) qui fleurissent surtout à partir de l'instauration définitive du régime libéral, au milieu des années 1830. Jean-René Aymes propose une typologie des fêtes religieuses et profanes à la même période, mettant en particulier en valeur la différence entre un monde rural symbole de la pérennité et un monde urbain plus sensible aux modes et aux changements politiques. Jean-François Botrel a choisi de son côté la description précise d'un de ces nouveaux espaces de sociabilité : le casino de la ville de León au milieu du $\mathrm{XIX}^{\mathrm{e}}$ siècle. L'article de Juan Francisco Fuentes est un des plus stimulants de l'ouvrage. Dans une description solidement problématisée, il montre le passage entre ce qu'il appelle la sociabilité censitaire et la sociabilité populaire. Si la «sphère publique bourgeoise" (pour reprendre la terminologie d'Habermäs) apparaît au XVIIIe siècle et conserve des caractéristiques qui lui sont propres au siècle suivant, les dernières années du siècle des Lumières voient l'émergence de la "publicité plebeyene "à la faveur de la guerre des Pyrénées (1793-1795). Le mouvement se développe par la suite pendant les phases libérales que connaît l'Espagne. La sociabilité populaire qui se construit alors est pour partie inspirée de la sociabilité bourgeoise (le journal...), pour partie inspirée de la sociabilité d'Ancien Régime (corporations...), pouvant donner localement le jour à un début de sociabilité ouvrière dès la fin des années 1830.

Ces phénomènes sont le reflet, mais aussi le moteur, de l'apparition d'une opinion publique, ce qui conduit les contemporains à s'interroger sur le sens à donner à ce terme dans la première moitié du XIXe siècle, et à dessiner les contours de ce qui doit être la vraie opinion publique, comme le montre l'article de Claude Morange. Deux études de cas illustrent la manière dont l'opinion s'est exprimée et les canaux qu'elle a empruntés à l'occasion de deux crises politiques majeures : celle de mai 1808 à Séville (Francisco Aguilar Piñal) et celle de 1823 (Irene Castells), au moment de l'invasion de la péninsule Ibérique par l'armée française des cent mille fils de Saint Louis.

Les articles sur la presse (d'Antonio Moliner, Agustín Martínez de las Heras, Ana Boned Colera, Maria Antonia Fernández) sont d'un grand intérêt, tout en restant très descriptifs et centrés sur les idées politiques, comme le sont la publication et le commentaire par María Rosa Saurín de la Iglesia de lettres.d'un des premiers libéraux galiciens.

Deux articles sont enfin consacrés à la réalité de la vie politique locale sous Isabelle II et sont tous deux excellents. Celui d'Andrés Barcala décrit par le menu l'émergence de familles d'origine commerçante dont sont issus des caciques de la fin du 
XIX ${ }^{c}$ siècle à Orihuela, dans la région de Valence. L'étude est passionnante car elle montre la diversité des trajectoires et des attitudes politiques de ces familles. Ainsi, les explications globalisantes à l'existence du caciquisme résistent mal à une telle étude de cas. Ce constat peut être aussi fait à propos de l'article de María Cruz Romeo sur les luttes politiques municipales dans la région de Valence. Ce travail montre que l'image monolithique et autoritaire des libéraux modérés est en grande partie à revoir. Il y a lutte politique à l'intérieur de cette famille, lutte qui entraîne une part importante des populations à l'échelle municipale et contribue à la diffusion de l'apprentissage de la politique moderne.

Ce livre, édité avec soin, est un instrument précieux pour comprendre l'apparition de la politique moderne dans l'Europe du XIXe siècle au travers du cas espagnol, mais aussi pour susciter une approche comparatiste du phénomène, approche qu'Alberto Gil Novales a lui-même souvent défendue et entretenue.

Jean-Philippe LUIS

Pascal M. Kitromilidis, La Révolution française et l'Europe du Sud-Est, Athènes, éd. Poreia, 2000, 194 p. (2e édition).

Pascal M. Kitromilidis, professeur de sciences politiques à l'Université d'Athènes, directeur du Centre des recherches néo-helléniques à la Fondation nationale de la Recherche, est l'auteur de nombreux ouvrages sur la Grèce du XVIIIe siècle (Rigas Vélestinlis. Théorie et action, 1998; Les Lumières néo-helléniques. Les idées politiques et sociales, 1996; La vision de la liberté dans la société grecque, 1992; ainsi qu'une édition grecque de l'œuvre de John Locke).

La première partie de ce nouvel ouvrage, la plus conséquente (plus d'une soixantaine de pages) pourrait presque se suffire à elle-même puisqu'elle évoque tous les thèmes qui seront abordés ultérieurement et permet d'exposer la problématique générale.

Lauteur commence par rendre hommage au grand historien roumain Nicolae Iorga qui, dans l'entre-deux-Guerres, fut l'un des premiers à vouloir rechercher les influences de la Révolution française dans le sud-est de l'Europe en dépassant le cadre territorial de l'Empire ottoman pour prendre davantage en compte le facteur humain (groupes sociaux, diasporas). P. Kitromilidis revient d'ailleurs sur la définition de cet espace géographique et culturel qui constitue, selon lui, le cadre historique de l'existence de l'hellénisme ou plus exactement de l'orthodoxie. Cet espace ne comprend pas seulement la péninsule balkanique au sud du Danube mais également les provinces méridionales de l'Empire des Habsbourg, les possessions vénitiennes de Dalmatie et d'Illyrie, Raguse ainsi que les ports de Venise et Trieste où sont concentrées d'importantes communautés balkaniques, enfin la côte occidentale de l'Asie Mineure et Chypre. Chronologiquement, l'étude ne saurait, d'après lui, être circonscrite à la décade 1789-1799 car l'éveil des mouvements nationaux se développe surtout pendant la période napoléonienne.

Reprenant un terme cher au professeur Michel Vovelle, il s'efforce ensuite de comprendre comment, dans cette entité géographique originale, s'est « forgée la mentalité révolutionnaire" (titre donné au chapitre), en y mesurant les échos des idées nouvelles.

Il distingue ainsi plusieurs niveaux d'influence et de réceptivité de l'idéologie révolutionnaire. Il y a en premier lieu les régions qui sont au contact direct avec l'administration française et qui subissent de plein fouet ses implications en terme de destruction de la féodalité et des privilèges, de réformes politiques et sociales. Telles sont les îles Ioniennes, occupées à deux reprises (de 1797 à 1799 , et de 1807 à 1814), et les provinces Illyriennes de Dalmatie, Istrie et Croatie (de 1806 à 1814). Il y a ensuite les représentants officiels et les agents secrets de la France révolutionnaire et napoléonienne qui, 
avec leurs réseaux de liaisons, véhiculent dans les grands centres urbains et les ports de l'Empire ottoman (à commencer par Constantinople) les attentes et les espoirs mais aussi les craintes d'un changement. Viennent enfin les intellectuels grecs de la diaspora, témoins oculaires des événements dans la capitale parisienne (Koraïs, Stamaty, Philippidis...), dont les écrits et les initiatives politiques présentent un intérêt particulier pour l'histoire comparée des idées.

À n'en pas douter, les masses paysannes de la société balkanique ne sont pas influencées par ce mouvement qui ne trouve résonance qu'auprès de certains milieux cultivés de l'aristocratie foncière (les Bogiares) et dans les classes urbaines des négociants. Les bases sociales de la réceptivité aux idées révolutionnaires sont donc assez faibles sans pour autant pouvoir être niées.

La seconde partie porte le titre : « La critique libérale de la Révolution française ». L'auteur propose une lecture du témoignage du savant Adamantios Koraiis (1748-1833) qui, dans sa Correspondance, «formule les inquiétudes psychologiques, spirituelles et politiques ainsi que les problématiques qui annoncent la critique libérale de la Révolution".

Installé à Paris au mois de mai 1788 pour y compléter ses recherches en vue d'une édition des textes Hypocratiques, Koraïs est, avec Constantin Stamaty, l'un des rares Grecs à être le témoin oculaire des événements révolutionnaires. Certains faits, dont P. Kitromilidis cite quelques passages intéressants, semblent l'avoir profondément marqué : ainsi par exemple, le transfert de la dépouille de Voltaire au Panthéon (juillet 1791), la substitution de l'acclamation traditionnelle « vive le roi » par la clameur révolutionnaire "vive la Nation ", ou encore la mort de Louis XVI. Ses attentes sont à leur comble en 1796 lorsque les Français entreprennent la conquête de l'Italie et des îles Ioniennes; mais l'auteur explique aussi que toutes les parties de la communauté orthodoxe et en particulier les classes dirigeantes locales, qui craignent l'extension de la Révolution, ne partagent pas l'enthousiasme de Koraïs.

P. Kitromilidis le situe politiquement dans le courant de pensée représentê en France par Condorcet et les Girondins dans les premières années de la Révolution, par les Idéologues et Mme de Staël sous le Directoire. Sa correspondance ultérieure avec Jérémy Bentham et Thomas Jefferson d'une part, et ses deux initiatives de traduction pour promouvoir les idées nouvelles dans la Grèce insurgée d'autre part (la Proclamation des Droits de l'homme et du citoyen et l'Essai sur les garanties individuelles de Pierre Claude François Daunou, l'un des plus fameux Idéologues) l'associent directement à la production littéraire libérale de la Révolution française.

Dans la troisième et dernière partie de son livre, P. Kitromilidis analyse ce qu'il appelle «le radicalisme balkanique » comme courant d'idées et comme action politique, qui préfigure et explique les événements révolutionnaires des années 1820 . Ce mouvement prend forme dans la dernière décennie du XVHII siècle et tire ses références idéologiques chez plusieurs auteurs des Lumières (Rousseau, Mably, Montesquieu) dont les œuvres sont traduites en grec dès le début de la Révolution.

Ce radicalisme est visible à trois niveaux. Dans le programme politique de Rigas Velestinli (1757-1798) (1) qui s'inspire de la Constitution française de 1793 et tranche avec les conceptions libérales de Koraïs; dans l'effervescence qui règne dans les milieux cultivés de la Grèce occidentale à la suite de l'occupation des Sept îles par les troupes françaises; enfin, dans les centres de la diaspora hellène en Europe centrale et orientale et dans certains ports de la Méditerranée (Livourne, Marseille), lesquels deviennent des points de contact du monde balkanique avec les idées nouvelles bien sûr (par le biais de

(1) Rigas est né dans la petite bourgade de Vélestino, non loin du port de Volos (Thessalie). Après la guerre d'indépendance de 1821, on ajouta à son nom le mot «Féraios ", ancien toponyme de Vélestino. 
la littérature et de la presse) mais aussi avec les pratiques politiques révolutionnaires. Vienne joue à cet égard un rôle majeur dans la diffusion à Bucarest, à Jassy, à Zagreb et à Raguse des brochures et libelles édités en grec et en serbo-croate ainsi que dans les tentatives de déstabilisation des principautés danubiennes et de Serbie sous domination ottomane.

Les deux manifestations les plus éclatantes de ce radicalisme précurseur et annonciateur des mouvements nationaux du début du XIXe siècle sont la conspiration de Martinovi à Vienne et à Budapest dans les années 1795-1796, à laquelle participent les Jacobins de Croatie et de Serbie, et la tentative de Rigas peu de temps après en 1798, initiatives qui se soldent l'une et l'autre par un échec. L'auteur montre très bien comment la répression qui s'ensuit contraint le mouvement révolutionnaire balkanique, qui n'est pas homogène, à déplacer son cadre d'action hors des frontières de l'Empire austro-hongrois et à s'organiser en sociétés secrètes dont nous ne connaissons que les noms. Les circonstances politiques dans les pays balkaniques font que ce radicalisme comme action politique, comme langage symbolique et comme vision de programme intervient à un moment où, en France, il est déjà vaincu après la chute des Jacobins et léchec de la "République de la Vertu " qu'ils voulaient créer.

L'un des intérêts majeurs de cet ouvrage est d'aborder chacune de ces questions en la replaçant dans une approche historiographique et de bien décrire le discours politique des précurseurs révolutionnaires grecs à partir du faible corpus de textes dont nous disposons. S'agissant d'une deuxième édition (la première a été publiée en 1990), P. Kitromilidis a pu aussi l'enrichir des nouveaux apports de la recherche.

Amaury FAIVRE D'ARCIER

Revue historique de Bordeaux et du département de la Gironde, $\mathrm{n}^{\circ}$ 1, troisième série, 2002 , $165 \mathrm{p}$.

Cette revue, née en 1908, a longtemps figuré au nombre des très bonnes revues provinciales. Assoupie, elle reprend vigueur grâce à la Fédération historique du Sud-Ouest et aux conservateurs des Archives municipales et départementales. Elle s'attachera à faire connaître, pour toutes les époques, les nouvelles approches de l'histoire locale. Le premier numéro porte sur Le cosmopolitisme bordelais. Les lecteurs des A.H.R.F. y trouveront en particulier des articles sur la colonie jacobite à Bordeaux au XVIII siècle (P. Clarke de Dromantin), sur Joseph Fenwick, Premier consul des États-Unis (S. Marzagalli), sur les Allemands au Grand-Théâtre de Bordeaux (A. Ruiz) et sur la Chine vue par des commerçants bordelais à la fin du XVIIIe siècle et au début du XIXe siècle (L. Bergès). Des comptes rendus de livres comme ceux de M. Figeac sur les Destins de la noblesse bordelaise de 1770 à 1830, de R. Coustet sur Bordeaux. Le temps de l'histoire. Architecture et urbanisme au XIXe siècle (1810-1914) ou de P. Guillaume sur les Hospices de Bordeaux au XIXe siècle, 1796-1855, s'ajoutent aux articles de la revue. Enfin une rubrique «Soutenances de thèses» publie le rapport de soutenance de M. C. L. Robin, "Le personnel politique du Libournais de Napoléon Bonaparte à Albert Lebrun ».

Jean-Paul BERTAUD 